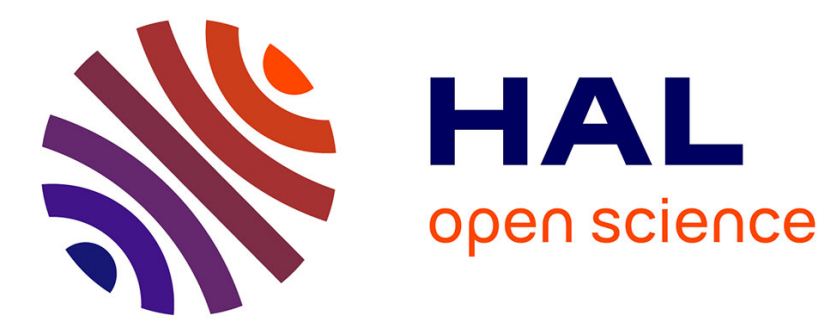

\title{
On Multivariate Extensions of Conditional-Tail-Expectation
}

Areski Cousin, Elena Di Bernardino

\section{To cite this version:}

Areski Cousin, Elena Di Bernardino. On Multivariate Extensions of Conditional-Tail-Expectation. 2013. hal-00877386

\author{
HAL Id: hal-00877386 \\ https://hal.science/hal-00877386
}

Preprint submitted on 28 Oct 2013

HAL is a multi-disciplinary open access archive for the deposit and dissemination of scientific research documents, whether they are published or not. The documents may come from teaching and research institutions in France or abroad, or from public or private research centers.
L'archive ouverte pluridisciplinaire HAL, est destinée au dépôt et à la diffusion de documents scientifiques de niveau recherche, publiés ou non, émanant des établissements d'enseignement et de recherche français ou étrangers, des laboratoires publics ou privés. 


\title{
On Multivariate Extensions of Conditional-Tail-Expectation
}

\author{
Areski Cousin, Elena Di Bernardino ${ }^{\dagger}$
}

28th October 2013

\begin{abstract}
In this paper, we introduce two alternative extensions of the classical univariate Conditional-TailExpectation (CTE) in a multivariate setting. The two proposed multivariate CTE are vector-valued measures with the same dimension as the underlying risk portfolio. As for the multivariate Valueat-Risk measures introduced in Cousin and Di Bernardino (2013), the lower-orthant CTE (resp. the upper-orthant CTE) is constructed from level sets of multivariate distribution functions (resp. of multivariate survival distribution functions). Contrary to allocation measures or systemic risk measures, these measures are also suitable for multivariate risk problems where risks are heterogenous in nature and cannot be aggregated together. Several properties have been derived. In particular, we show that the proposed multivariate CTE-s satisfy natural extensions of the positive homogeneity property, the translation invariance property and the comonotonic additivity property. Comparison between univariate risk measures and components of multivariate CTE are provided. We also analyze how these measures are impacted by a change in marginal distributions, by a change in dependence structure and by a change in risk level. Sub-additivity of the proposed multivariate CTE-s is provided under the assumption that all components of the random vectors are independent. Illustrations are given in the class of Archimedean copulas.
\end{abstract}

Keywords: Multivariate risk measures, Level sets of distribution functions, Multivariate probability integral transformation, Stochastic orders, Copulas and dependence.

\section{Introduction}

As illustrated by the recent financial turmoil, risks are strongly interconnected. Consequently, risk quantification in multivariate settings has recently been the subject of great interest. Much research has been devoted to construction of risk measures that account both for marginal effects and dependence between risks.

In the literature, several generalizations of the classical univariate Conditional-Tail-Expectation (CTE) have been proposed, mainly using as conditioning events the total risk or some extreme risks. These measures can be used as capital allocation rules for financial institutions. The aim is to find the contribution of each subsidiary (or risk category) to the total economic capital. As can be seen in Scaillet (2004) and Tasche (2008), the Euler or Shapley-Aumann allocation rule associated with a particular univariate risk measure (such as VaR or CTE) involves the dependence structure between marginal and aggregated risks. More formally, let $\mathbf{X}=\left(X_{1}, \ldots, X_{d}\right)$ represent the risk exposures of a given financial institution, where, for any $i=1, \ldots, d$, the component $X_{i}$ denotes the marginal risk (usually claim or loss) associated with the underlying entity $i$ (the latter could be, for instance, a subsidiary, an operational branch or a risk category). Then, the sum $S=X_{1}+\cdots+X_{d}$ corresponds to the company aggregated risk, whereas $X_{(1)}=\min \left\{X_{1}, \ldots, X_{d}\right\}$ and $X_{(d)}=\max \left\{X_{1}, \ldots, X_{d}\right\}$ are the extreme risks. In capital allocation problems, we are not only interested in the "stand-alone" risk measures $\operatorname{CTE}_{\alpha}\left(X_{i}\right)=\mathbb{E}\left[X_{i} \mid X_{i}>Q_{X_{i}}(\alpha)\right]$,

\footnotetext{
*Université de Lyon, Université Lyon 1, ISFA, Laboratoire SAF, 50 avenue Tony Garnier, 69366 Lyon, France, Tel.: +33437287439, areski.cousin@univ-lyon1.fr, http://www.acousin.net/.

${ }^{\dagger}$ CNAM, Paris, Département IMATH, 292 rue Saint-Martin, Paris Cedex 03, France. elena.di_bernardino@cnam.fr. http://isfaserveur.univ-lyon1.fr/ẽlena.dibernardino/.
} 
where $Q_{X_{i}}(\alpha)=\inf \left\{x \in \mathbb{R}_{+}: F_{X_{i}}(x) \geq \alpha\right\}$ is the univariate quantile function of $X_{i}$ at risk level $\alpha$, but also in

$$
\begin{gathered}
\operatorname{CTE}_{\alpha}^{\text {sum }}\left(X_{i}\right)=\mathbb{E}\left[X_{i} \mid S>Q_{S}(\alpha)\right], \\
\operatorname{CTE}_{\alpha}^{\min }\left(X_{i}\right)=\mathbb{E}\left[X_{i} \mid X_{(1)}>Q_{X_{(1)}}(\alpha)\right], \\
\operatorname{CTE}_{\alpha}^{\max }\left(X_{i}\right)=\mathbb{E}\left[X_{i} \mid X_{(d)}>Q_{X_{(d)}}(\alpha)\right],
\end{gathered}
$$

for $i=1, \ldots, d$. The interested reader is referred to Cai and Li (2005) for further details. Explicit formula for $\operatorname{CTE}_{\alpha}^{\text {sum }}\left(X_{i}\right)$ are provided in Landsman and Valdez (2003) in the case of elliptic distribution functions, Cai and Li (2005) for phase-type distributions and in Bargès et al. (2009) for Fairlie-Gumbel-Morgenstern family of copulas. Furthermore, we recall that $\mathrm{CTE}_{\alpha}^{\text {sum }}\left(X_{i}\right)$ corresponds to the "Euler allocation rule" associated with the univariate CTE (see, e.g., Tasche (2008)).

Another problem which recently receives a great interest is the construction of systemic risk measures. One of the proposed measure is the Marginal Expected Shortfall (MES) defined as the expected loss on its equity return $(X)$ conditional on the occurrence of a loss in the aggregated return of the financial sector $(Y)$, i.e.,

$$
\operatorname{MES}_{\alpha}(X)=\mathbb{E}\left[X \mid Y>Q_{Y}(\alpha)\right],
$$

where $Q_{Y}(\alpha)$ is the $(\alpha)$-th quantile of the distribution of $Y$. The MES of a financial institution aims at detecting which firms in the economy are the more vulnerable in case of a global financial distress. On mathematical grounds, this measure is similar to the allocation measure CTE ${ }^{\text {sum }}$. The interested reader is referred to Acharya et al. (2010) or Brownlees and Engle (2012) for more details. Cai et al. (2013) propose a non-parametric estimator of the MES using extreme value theory. The CoVaR (conditional VaR) of company $i$ is instead given by

$$
\operatorname{CoVaR}_{\alpha}^{i}(X)=\operatorname{VaR}_{\alpha}\left(S \mid X \geq \operatorname{VaR}_{\alpha}(X)\right) .
$$

As opposed to the MES, the CoVaR is constructed in order to identify which firms in the economy have a great importance in terms of systemic risk (see Adrian and Brunnermeier (2011) or Mainik and Schaanning (2012)).

However, the previous risk measures are not suitable for multivariate risk problem where risks are heterogeneous in nature and thus cannot be aggregated together or even compared. This is the case for instance for risks which are difficult to expressed under the same numéraire or when one has to deal with non-monetary risks or exogenous risks. The literature which deals with risk measures for intrinsically multivariate problems can be divided in two categories. The first group of papers are interested in extending classical univariate axioms to different multivariate settings (see for instance Jouini et al. (2004), Burgert and Rüschendorf (2006), Rüschendorf (2006), Cascos and Molchanov (2007), Hamel and Heyde (2010), Ekeland et al. (2012)). One of the objectives is to derive theoretical representation of risk measures. This is done without proposing tractable constructions for the axiom-consistent multivariate measures. Another group of papers investigates different generalizations of the concept of quantiles in a multivariate setting. Unsurprisingly, the main difficulty regarding multivariate generalizations of quantile-based risk measures (as the VaR and the CTE) is the fact that vector preorders are, in general, partial preorders. Then, what can be considered in a context of multidimensional portfolios as the analogous of a "worst case" scenario and a related "tail distribution"? For example, Massé and Theodorescu (1994) define multivariate $\alpha$-quantiles for bivariate distribution as the intersection of half-planes whose the distribution is at least equal to $\alpha$. Koltchinskii (1997) provides a general treatment of multivariate quantiles as inversions of mappings. Another approach is to use geometric quantiles (see, for example, Chaouch et al. (2009)). Along with the geometric quantile, the notion of depth function has been developed in recent years to characterize the quantile of multidimensional distribution functions (for further details see, for instance, Chauvigny et al. (2011)). We refer to Serfling (2002) for a review of multivariate quantiles.

When it turns to generalize the Value-at-Risk measure, Embrechts and Puccetti (2006), Nappo and Spizzichino (2009), Prékopa (2012) use the notion of quantile curve but these papers do not investigate whether these measures are compatible with some desirable axioms. Moreover, the proposed risk measures are hyperspaces and thus quantify a vector of risks with an infinite number of points. Contrarily 
to the latter approach, the multivariate Conditional-Tail-Expectation proposed in this paper quantifies multivariate risks in a more parsimonious and synthetic way. This feature can be relevant for operational applications since it can ease discrimination between portfolio of risks. Lee and Prékopa (2012) introduce a real-valued measure of multivariate risks which also bears on quantile curves but the proposed measure relies on a somehow arbitrary convex combination.

We propose two vector-valued extensions of the univariate Conditional-Tail-Expectation. The lowerorthant CTE of a random vector $X$ (introduced by Di Bernardino et al. (2013) in a bivariate setting) is defined as the conditional expectation of $X$ given that the latter is located in the $\alpha$-upper level set of its distribution function. The upper-orthant CTE of $X$ is defined as the conditional expectation of $X$ given that the latter is in the $(1-\alpha)$-lower level set of its survival function. Several properties have been derived. We provide an integral representation of the proposed measures in terms of the multivariate VaR introduced in Cousin and Di Bernardino (2013) and we show that the proposed multivariate CTE-s satisfy natural extensions of the positive homogeneity property, the translation invariance property and the comonotonic additivity property. We show that the proposed measures are sub-additive for independent vectors with independent components. We also provide comparisons between univariate risk measures and components of the proposed multivariate CTE. We analyze how these measures are impacted by a change in marginal distributions, by a change in dependence structure and by a change in risk level.

The paper is organized as follows. In Section 2, we give the definition of the lower-orthant and the upper-orthant Conditional-Tail-Expectation measures. We then show that these measures satisfy multivariate extensions of Artzner et al. (1999)'s invariance properties (see Section 2.1). Illustrations in some Archimedean copula cases are presented in Section 2.2. We also compare the components of these multivariate CTE measures with the associated univariate VaR, the associated univariate CTE and with the multivariate lower-orthant and upper-orthant VaR previously introduced by Cousin and Di Bernardino (2013) (see Section 2.3). The behavior of our CTE-s with respect to a change in marginal distributions, a change in dependence structure and a change in risk level $\alpha$ is discussed respectively in Sections 2.4, 2.5 and 2.6. The conclusion discusses open problems and possible directions for future work.

\section{Multivariate generalization of the Conditional-Tail-Expectation measure}

As in the univariate case, the multivariate VaR introduced in Cousin and Di Bernardino (2013) does not give any information regarding the upper tail of the loss distribution function and especially its degree of thickness above the VaR threshold. In an univariate setting, the problem has been overcome by considering for instance the Conditional-Tail-Expectation (CTE) risk measure ${ }^{1}$, which is defined as the conditional expectation of losses given that the latter exceed VaR. Following Artzner et al. (1999), the CTE at level $\alpha$ for a distribution function $F$ (or survival function $\bar{F}$ ) is given by

$$
\mathrm{CTE}_{\alpha}(X):=\mathbb{E}\left[X \mid X \geq \operatorname{VaR}_{\alpha}(X)\right],
$$

where $\operatorname{VaR}_{\alpha}(X)$ is the univariate Value-at-Risk defined by

$$
\operatorname{VaR}_{\alpha}(X):=\inf \{x \in \mathbb{R}: F(x) \geq \alpha\}=\inf \{x \in \mathbb{R}: \bar{F}(x) \leq 1-\alpha\} .
$$

Since the sets $\left\{X \geq \operatorname{VaR}_{\alpha}(X)\right\},\{F(X) \geq \alpha\}$ and $\{\bar{F}(X) \leq 1-\alpha\}$ correspond to the same event in a univariate setting, the CTE can alternatively be defined ${ }^{2}$ as

$$
\mathrm{CTE}_{\alpha}(X):=\mathbb{E}[X \mid F(X) \geq \alpha]=\mathbb{E}[X \mid \bar{F}(X) \leq 1-\alpha]
$$

The CTE can then be viewed as the conditional expectation of $X$ given that $X$ falls into the $\alpha$-lower-level set of its distribution function $\underline{L}(\alpha):=\left\{x \in \mathbb{R}_{+}: F(x) \geq \alpha\right\}$ or equivalently in the $(1-\alpha)$-upper-level set

\footnotetext{
${ }^{1}$ This measure is also called Tail Conditional Expectation. As far as continuous distribution functions are considered, the CTE measure is coherent in the sense of Artzner's axioms and it coincides with the Expected Shortfall or Tail VaR.

${ }^{2}$ Note that this definition does not depend on VaR.
} 
of its survival function $\bar{L}(\alpha):=\left\{x \in \mathbb{R}_{+}: \bar{F}(x) \leq 1-\alpha\right\}$. This definition can naturally be extended in higher dimension, keeping in mind that the two previous sets $\underline{L}(\alpha)$ and $\bar{L}(\alpha)$ are different in general as soon as the dimension $d$ of the underlying risk vector is greater or equal to 2 . In analogy with Embrechts and Puccetti (2006) notations, we will denote by CTE the lower-orthant Conditional-Tail-Expectation based on level sets of the underlying distribution function (distrubution function measures mass of probabilities in lower-orthant regions) and by $\overline{\mathrm{CTE}}$ the upper-orthant Conditional-Tail-Expectation based on level sets of the underlying survival function (survival function measures mass of probabilities in upper-orthant regions).

Assumption 2.1. In the following, we will consider non-negative absolutely-continuous random vector ${ }^{3}$ $\boldsymbol{X}=\left(X_{1}, \ldots, X_{d}\right)$ (with respect to Lebesgue measure $\lambda$ on $\mathbb{R}^{d}$ ) with partially increasing multivariate distribution function ${ }^{4} F$ and such that $\mathbb{E}\left(X_{i}\right)<\infty$, for $i=1, \ldots, d$. These conditions will be called regularity conditions.

Definition 2.1 (Multivariate lower-orthant and upper-orthant Conditional-Tail-Expectation). Consider a random vector $\boldsymbol{X}=\left(X_{1}, \ldots, X_{d}\right)$ with distribution function $F$ and survival function $\bar{F}$, such that $\mathbb{E}\left[X_{i}\right]$ is finite for all $i=1, \ldots, d$. For $\alpha \in(0,1)$, we define the lower-orthant Conditional-Tail-Expectation at probability level $\alpha$ by

$$
\underline{\mathrm{CTE}}_{\alpha}(\boldsymbol{X})=\mathbb{E}[\boldsymbol{X} \mid F(\boldsymbol{X}) \geq \alpha]=\left(\begin{array}{c}
\mathbb{E}\left[X_{1} \mid F(\boldsymbol{X}) \geq \alpha\right] \\
\vdots \\
\mathbb{E}\left[X_{d} \mid F(\boldsymbol{X}) \geq \alpha\right]
\end{array}\right),
$$

and the upper-orthant Conditional-Tail-Expectation at probability level $\alpha$ by

$$
\overline{\mathrm{CTE}}_{\alpha}(\boldsymbol{X})=\mathbb{E}[\boldsymbol{X} \mid \bar{F}(\boldsymbol{X}) \leq 1-\alpha]=\left(\begin{array}{c}
\mathbb{E}\left[X_{1} \mid \bar{F}(\boldsymbol{X}) \leq 1-\alpha\right] \\
\vdots \\
\mathbb{E}\left[X_{d} \mid \bar{F}(\boldsymbol{X}) \leq 1-\alpha\right]
\end{array}\right)
$$

Remark that the lower-orthant CTE is a multivariate generalization of the bivariate Conditional-TailExpectation previously introduced by Di Bernardino et al. (2013).

From now on, we denote by $\underline{\mathrm{CTE}}_{\alpha}^{1}(\mathbf{X}), \ldots, \underline{\mathrm{CTE}}_{\alpha}^{d}(\mathbf{X})$ the components of the vector $\underline{\mathrm{CTE}}_{\alpha}(\mathbf{X})$ and by $\overline{\mathrm{CTE}}_{\alpha}^{1}(\mathbf{X}), \ldots, \overline{\mathrm{CTE}}_{\alpha}^{d}(\mathbf{X})$ the components of the vector $\overline{\mathrm{CTE}}_{\alpha}(\mathbf{X})$.

Note that if $\mathbf{X}$ is an exchangeable random vector, $\underline{\mathrm{CTE}}_{\alpha}^{i}(\mathbf{X})=\underline{\mathrm{CTE}}_{\alpha}^{j}(\mathbf{X})$ and $\overline{\mathrm{CTE}}_{\alpha}^{i}(\mathbf{X})=\overline{\mathrm{CTE}}_{\alpha}^{j}(\mathbf{X})$ for any $i, j=1, \ldots, d$. Furthermore, given a univariate random variable $X, \mathbb{E}\left[X \mid F_{X}(X) \geq \alpha\right]=$ $\mathbb{E}\left[X \mid \bar{F}_{X}(X) \leq 1-\alpha\right]=\mathrm{CTE}_{\alpha}(\mathrm{X})$, for all $\alpha$ in $(0,1)$. Hence, lower-orthant and upper-orthant ConditionalTail-Expectation coincide with the usual CTE for (univariate) random variables.

Let us remark that, under the regularity assumption, the multivariate lower-orthant (resp. upper-orthant) Conditional-Tail-Expectation can be represented as an integral transformation of the multivariate lowerorthant VaR (resp. upper-orthant $\overline{\mathrm{VaR}}$ ) introduced by Cousin and Di Bernardino (2013), i.e.,

$$
\underline{\mathrm{CTE}}_{\alpha}^{i}(\mathbf{X})=\frac{1}{1-K(\alpha)} \int_{\alpha}^{1} \underline{\operatorname{VaR}}_{\gamma}^{i}(\mathbf{X}) K^{\prime}(\gamma) \mathrm{d} \gamma,
$$

and

$$
\overline{\mathrm{CTE}}_{\alpha}^{i}(\mathbf{X})=\frac{1}{\widehat{K}(1-\alpha)} \int_{\alpha}^{1} \overline{\operatorname{VaR}}_{\gamma}^{i}(\mathbf{X}) \widehat{K}^{\prime}(1-\gamma) \mathrm{d} \gamma,
$$

\footnotetext{
${ }^{3}$ We restrict ourselves to $\mathbb{R}_{+}^{d}$ because, in our applications, components of $d$-dimensional vectors correspond to random losses and are then valued in $\mathbb{R}_{+}$.

${ }^{4} \mathrm{~A}$ function $F\left(x_{1}, \ldots, x_{d}\right)$ is partially increasing on $\mathbb{R}_{+}^{d} \backslash(0, \ldots, 0)$ if the functions of one variable $g(\cdot)=$ $F\left(x_{1}, \ldots, x_{j-1}, \cdot, x_{j+1}, \ldots, x_{d}\right)$ are increasing. About properties of partially increasing multivariate distribution functions we refer the interested reader to Rossi (1973), Tibiletti (1991).
} 
where $K$ is the Kendall distribution of $X$, i.e., $K(x)=\mathbb{P}(F(\mathbf{X}) \leq x)$, for all $x$ in $(0,1)$ and $\widehat{K}$ is its "upper-orthant" Kendall distribution, i.e., $\widehat{K}(x)=\mathbb{P}(\bar{F}(\mathbf{X}) \leq x)$, for all $x$ in $(0,1)$. Formula (8) and (9) will be useful in Proposition 2.8 and Corollary 2.3 below.

Remark that the existence of densities $K^{\prime}$ and $\widehat{K}^{\prime}$ that appears in Equations (8)-(9)) is guaranteed by the regularity conditions (for further details, see Proposition 1 in Imlahi et al. (1999) or Proposition 4 in Chakak and Ezzerg (2000)). The interested reader is also referred to Cousin and Di Bernardino (2013).

\subsection{Invariance properties}

In the present section, the aim is to analyze the lower-orthant and the upper-orthant CTE introduced in Definition 2.1 in terms of classical invariance properties of risk measures (we refer the interested reader to Artzner et al. (1999)). In analogy with Section 2.1 in Cousin and Di Bernardino (2013), we now introduce the following results (Proposition 2.1 and Corollary 2.1) that will be useful in order to prove invariance properties of our risk measures.

Proposition 2.1. Let the function $h$ be such that $h\left(x_{1}, \ldots, x_{d}\right)=\left(h_{1}\left(x_{1}\right), \ldots, h_{d}\left(x_{d}\right)\right)$.

- If $h_{1}, \ldots, h_{d}$ are non-decreasing functions, then the following relations hold

$$
\underline{\mathrm{CTE}}_{\alpha}^{i}(h(\boldsymbol{X}))=\mathbb{E}\left[h_{i}\left(X_{i}\right) \mid F_{\boldsymbol{X}}(\boldsymbol{X}) \geq \alpha\right], \quad i=1, \ldots, d
$$

and

$$
\overline{\mathrm{CTE}}_{\alpha}^{i}(h(\boldsymbol{X}))=\mathbb{E}\left[h_{i}\left(X_{i}\right) \mid \bar{F}_{\boldsymbol{X}}(\boldsymbol{X}) \leq 1-\alpha\right], \quad i=1, \ldots, d .
$$

- If $h_{1}, \ldots, h_{d}$ are non-increasing functions, then the following relations hold

$$
\underline{\mathrm{CTE}}_{\alpha}^{i}(h(\boldsymbol{X}))=\mathbb{E}\left[h_{i}\left(X_{i}\right) \mid \bar{F}_{\boldsymbol{X}}(\boldsymbol{X}) \geq \alpha\right], \quad i=1, \ldots, d
$$

and

$$
\overline{\mathrm{CTE}}_{\alpha}^{i}(h(\boldsymbol{X}))=\mathbb{E}\left[h_{i}\left(X_{i}\right) \mid F_{\boldsymbol{X}}(\boldsymbol{X}) \leq 1-\alpha\right], \quad i=1, \ldots, d .
$$

Proof: From Definition 2.1, $\underline{\mathrm{CTE}}_{\alpha}^{i}(h(\mathbf{X}))=\mathbb{E}\left[h_{i}\left(X_{i}\right) \mid F_{h(\mathbf{X})}(h(\mathbf{X})) \geq \alpha\right]$, for $i=1, \ldots, d$. Since

$$
F_{h(\mathbf{X})}\left(y_{1}, \ldots, y_{d}\right)= \begin{cases}F_{\mathbf{X}}\left(h^{-1}\left(y_{1}\right), \ldots, h^{-1}\left(y_{d}\right)\right), & \text { if } h_{1}, \ldots, h_{d} \text { are non-decreasing functions } \\ \bar{F}_{\mathbf{X}}\left(h^{-1}\left(y_{1}\right), \ldots, h^{-1}\left(y_{d}\right)\right), & \text { if } h_{1}, \ldots, h_{d} \text { are non-increasing functions }\end{cases}
$$

then we obtain the result.

Finally, we can state the following result that proves positive homogeneity and translation invariance for our measures.

Proposition 2.2. For $\alpha \in(0,1)$, the multivariate upper-orthant and lower-orthant Conditional-TailExpectation satisfy the following properties:

Positive Homogeneity: $\quad \forall \boldsymbol{c}=\left(c_{1}, \ldots, c_{d}\right)^{\prime} \in \mathbb{R}_{+}^{d}$,

$$
\begin{aligned}
& \underline{\mathrm{CTE}}_{\alpha}\left(c_{1} X_{1}, \ldots, c_{d} X_{d}\right)=\left(c_{1} \underline{\mathrm{CTE}}_{\alpha}^{1}(\boldsymbol{X}), \ldots, c_{d} \underline{\mathrm{CTE}}_{\alpha}^{d}(\boldsymbol{X})\right)^{\prime} \\
& \overline{\mathrm{CTE}}_{\alpha}\left(c_{1} X_{1}, \ldots, c_{d} X_{d}\right)=\left(c_{1} \overline{\mathrm{CTE}}_{\alpha}^{1}(\boldsymbol{X}), \ldots, c_{d} \overline{\mathrm{CTE}}_{\alpha}^{d}(\boldsymbol{X})\right)^{\prime}
\end{aligned}
$$

Translation Invariance: $\forall \boldsymbol{c} \in \mathbb{R}_{+}^{d}$,

$$
\underline{\mathrm{CTE}}_{\alpha}(\boldsymbol{c}+\boldsymbol{X})=\boldsymbol{c}+\underline{\mathrm{CTE}}_{\alpha}(\boldsymbol{X}), \quad \overline{\mathrm{CTE}}_{\alpha}(\boldsymbol{c}+\boldsymbol{X})=\boldsymbol{c}+\overline{\mathrm{CTE}}_{\alpha}(\boldsymbol{X})
$$

The proof comes down from Proposition 2.1. 
Remark 1. i) In the univariate as well in the multivariate setting, a risk measure may be interpreted as the riskiness of a portfolio or the amount of capital that should be added to a portfolio with a given loss, so that the portfolio can then be deemed acceptable. In this respect, the translation invariance property for (multivariate) risk measures are necessary if the risk-capital interpretation we stated above is to make sense. The homogeneity property is often motivated by a change of currency argument: the amount of required capital in order to manager risks should be independent of the currency in which it is expressed (e.g. see Artzner et al. (1999)). For these reasons, the invariance properties proved in Proposition 2.2 play a central role in practical applications.

ii) It is straightforward to show that the capital allocation measures presented in (1)-(3) do not satisfy the invariance properties stated in Proposition 2.2 as soon as the components of $\mathbf{c}=\left(c_{1}, \ldots, c_{d}\right)$ are all different. In that sense, these measures may not be suitable for intrinsically multivariate problem where risks cannot be expressed under the same numéraire.

Remark 2. For $\alpha=0$, using Definition 2.1, we obtain

$$
\underline{\mathrm{CTE}}_{0}(\boldsymbol{X})=\overline{\mathrm{CTE}}_{0}(\boldsymbol{X})=\left(\begin{array}{c}
\mathbb{E}\left[X_{1}\right] \\
\vdots \\
\mathbb{E}\left[X_{d}\right]
\end{array}\right)=\mathbb{E}[\boldsymbol{X}]
$$

Then, as in the univariate case, the multivariate lower-orthant and upper-orthant CTE-s are equal to the expected value of the underlying random vector for $\alpha=0$.

Since these two new measures are not the same in general for dimension greater or equal to 2 , we also provide some connections between $\underline{\mathrm{CTE}}$ and $\overline{\mathrm{CTE}}$. From Proposition 2.1 one can obtain the following property which links the multivariate upper-orthant Conditional-Tail-Expectation and the lower-orthant one.

Corollary 2.1. Let $h$ be a linear function such that $h\left(x_{1}, \ldots, x_{d}\right)=\left(h_{1}\left(x_{1}\right), \ldots, h_{d}\left(x_{d}\right)\right)$.

- If $h_{1}, \ldots, h_{d}$ are non-decreasing functions then it holds that

$$
\underline{\mathrm{CTE}}_{\alpha}(h(\boldsymbol{X}))=h\left(\underline{\mathrm{CTE}}_{\alpha}(\boldsymbol{X})\right) \quad \text { and } \quad \overline{\mathrm{CTE}}_{\alpha}(h(\boldsymbol{X}))=h\left(\overline{\mathrm{CTE}}_{\alpha}(\boldsymbol{X})\right) .
$$

- If $h_{1}, \ldots, h_{d}$ are non-increasing functions then it holds that

$$
\underline{\mathrm{CTE}}_{\alpha}(h(\boldsymbol{X}))=h\left(\frac{\mathbb{E}[X]}{1-\widehat{K}(\alpha)}-\frac{\widehat{K}(\alpha)}{1-\widehat{K}(\alpha)} \overline{\operatorname{CTE}}_{1-\alpha}(\boldsymbol{X})\right)
$$

and

$$
\overline{\mathrm{CTE}}_{\alpha}(h(\boldsymbol{X}))=h\left(\frac{\mathbb{E}[X]}{K(1-\alpha)}-\frac{1-K(1-\alpha)}{K(1-\alpha)} \underline{\mathrm{CTE}}_{1-\alpha}(\boldsymbol{X})\right) .
$$

Example 1. If $X=\left(X_{1}, \ldots, X_{d}\right)$ is a random vector with uniform margins and if, for all $i=1, \ldots, d$, we consider the functions $h_{i}$ such that $h_{i}(x)=1-x, x \in[0,1]$, then from Corollary 2.1, we get

$$
\overline{\mathrm{CTE}}_{\alpha}^{i}(\boldsymbol{X})=\frac{1}{\widehat{K}(1-\alpha)}\left[\frac{1}{2}-(1-\widehat{K}(1-\alpha))\left(1-\underline{\mathrm{CTE}}_{1-\alpha}^{i}(\boldsymbol{1}-\boldsymbol{X})\right)\right]
$$

for all $i=1, \ldots, d$, where $1-\boldsymbol{X}=\left(1-X_{1}, \ldots, 1-X_{d}\right)$. Note that $\widehat{K}$ is the Kendall distribution function associated with the vector $\mathbf{1}-\boldsymbol{X}$. Additionally, if $\boldsymbol{X}$ and $\mathbf{1}-\boldsymbol{X}$ have the same distribution function, then $X$ is invariant in law by central symmetry and the following relation holds:

$$
\overline{\mathrm{CTE}}_{\alpha}^{i}(\boldsymbol{X})=\frac{1}{K(1-\alpha)}\left[\frac{1}{2}-(1-K(1-\alpha))\left(1-\underline{\mathrm{CTE}}_{1-\alpha}^{i}(\boldsymbol{X})\right)\right]
$$

For instance, this property is satisfied for vector $\mathbf{X}$ which follows an elliptical or a Frank copula structure. 
We now show that, as in the univariate setting, the lower-orthant and the upper-orthant CTE-s are additive for $\pi$-comonotonic couple of random vectors as defined in Puccetti and Scarsini (2010).

Definition 2.2. A couple $(\mathbf{X}, \mathbf{Y})$ of d-dimensional random vectors is said to be $\pi$-comonotonic if there exists a d-dimensional random vector $\mathbf{Z}$ and non-decreasing functions $f_{1}, \ldots, f_{d}, g_{1}, \ldots, g_{d}$ such that

$$
(\mathbf{X}, \mathbf{Y}) \stackrel{d}{=}\left(\left(f_{1}\left(Z_{1}\right), \ldots, f_{d}\left(Z_{d}\right)\right),\left(g_{1}\left(Z_{1}\right), \ldots, g_{d}\left(Z_{d}\right)\right)\right)
$$

Proposition 2.3. Let $(\mathbf{X}, \mathbf{Y})$ be a $\pi$-comonotonic couple of random vectors, then

$$
\underline{\mathrm{CTE}}_{\alpha}(\mathbf{X}+\mathbf{Y})=\underline{\mathrm{CTE}}_{\alpha}(\mathbf{X})+\underline{\mathrm{CTE}}_{\alpha}(\mathbf{Y}), \quad \overline{\mathrm{CTE}}_{\alpha}(\mathbf{X}+\mathbf{Y})=\overline{\mathrm{CTE}}_{\alpha}(\mathbf{X})+\overline{\mathrm{CTE}}_{\alpha}(\mathbf{Y})
$$

Proof: We focus on the lower-orthant CTE. Similar arguments apply for the upper-orthant CTE. Let $\mathbf{X}=\left(X_{1}, \ldots, X_{d}\right)$ and $\mathbf{Y}=\left(Y_{1}, \ldots, Y_{d}\right)$ be two $\pi$-comonotonic random vectors. There exits a random vector $\mathbf{Z}=\left(Z_{1}, \ldots, Z_{d}\right)$ such that, for any $i=1, \ldots, d, X_{i}=f_{i}\left(Z_{i}\right)$ and $Y_{i}=g_{i}\left(Z_{i}\right)$ where $f_{i}$ and $g_{i}$ are non-decreasing functions. Let $f$ be the function defined by $f\left(x_{1}, \ldots, x_{d}\right)=\left(f_{1}\left(x_{1}\right), \ldots, f_{d}\left(x_{d}\right)\right), g$ be the function defined by $g\left(x_{1}, \ldots, x_{d}\right)=\left(g_{1}\left(x_{1}\right), \ldots, g_{d}\left(x_{d}\right)\right)$ and $h$ be the function defined by $h\left(x_{1}, \ldots, x_{d}\right)=$ $\left(h_{1}\left(x_{1}\right), \ldots, h_{d}\left(x_{d}\right)\right)$ where $h_{i}:=f_{i}+g_{i}, i=1, \ldots, d$. The function $h_{i}, i=1, \ldots, d$ are non-decreasing as a sum of non-decreasing functions and $\mathbf{X}+\mathbf{Y}=h(\mathbf{Z})$. We obtain from Prop. 2.1 that, for any $i=1, \ldots, d$, $\underline{\mathrm{CTE}}_{\alpha}^{i}(\mathbf{X}+\mathbf{Y})=\mathbb{E}\left[h_{i}\left(Z_{i}\right) \mid F_{\mathbf{Z}}(\mathbf{Z}) \geq \alpha\right]=\mathbb{E}\left[f_{i}\left(Z_{i}\right) \mid F_{\mathbf{Z}}(\mathbf{Z}) \geq \alpha\right]+\mathbb{E}\left[g_{i}\left(Z_{i}\right) \mid F_{\mathbf{Z}}(\mathbf{Z}) \geq \alpha\right]$ where $F_{\mathbf{Z}}$ denotes the distribution function of $\mathbf{Z}$. Eventually, $\mathbb{E}\left[f_{i}\left(Z_{i}\right) \mid F_{\mathbf{Z}}(\mathbf{Z}) \geq \alpha\right]=\mathbb{E}\left[f_{i}\left(Z_{i}\right) \mid F_{\mathbf{f}(\mathbf{Z})}(\mathbf{f}(\mathbf{Z})) \geq \alpha\right]$

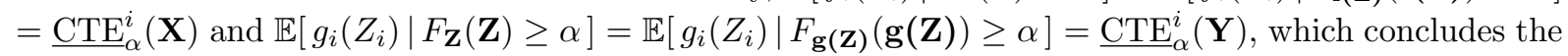
proof.

Remark 3. The previous proposition also holds for the lower-othant VaR and the upper-orthant VaR introduced in Cousin and Di Bernardino (2013).

We now prove that the proposed multivariate measures are subadditive for independent random vectors with independent components.

Proposition 2.4. Let $\boldsymbol{X}$ and $\boldsymbol{Y}$ be two d-dimensional independent random vectors with finite expectations for each marginal. If all components in $\boldsymbol{X}$ and $\boldsymbol{Y}$ are independent, then the lower-orthant ConditionalTail-Expectation $\underline{\mathrm{CTE}}$ is such that

$$
\underline{\mathrm{CTE}}_{\alpha}(\boldsymbol{X}+\boldsymbol{Y}) \leq \underline{\mathrm{CTE}}_{\alpha}(\boldsymbol{X})+\underline{\mathrm{CTE}}_{\alpha}(\boldsymbol{Y}), \text { for } \alpha \in(0,1) .
$$

The same result holds for the lower-orthant Conditional-Tail-Expectation $\overline{\mathrm{CTE}}$.

Proof: It is equivalent to prove that, for all $i=1, \ldots, d$ and for all $\alpha \in(0,1)$,

$$
\mathbb{E}\left[X_{i}+Y_{i} \mid F_{\mathbf{X}}+\mathbf{Y}(\mathbf{X}+\mathbf{Y}) \geq \alpha\right] \leq \mathbb{E}\left[X_{i} \mid F_{\mathbf{X}}(\mathbf{X}) \geq \alpha\right]+\mathbb{E}\left[Y_{i} \mid F_{\mathbf{Y}}(\mathbf{Y}) \geq \alpha\right]
$$

Equation (12) can be written as:

$$
\frac{\mathbb{E}\left[X_{i}+Y_{i}, F_{\mathbf{X}}+\mathbf{Y}(\mathbf{X}+\mathbf{Y}) \geq \alpha\right]}{\mathbb{P}\left[F_{\mathbf{X}}+\mathbf{Y}(\mathbf{X}+\mathbf{Y}) \geq \alpha\right]} \leq \frac{\mathbb{E}\left[X_{i}, F_{\mathbf{X}}(\mathbf{X}) \geq \alpha\right]}{\mathbb{P}\left[F_{\mathbf{X}}(\mathbf{X}) \geq \alpha\right]}+\frac{\mathbb{E}\left[Y_{i}, F_{\mathbf{Y}}(\mathbf{Y}) \geq \alpha\right]}{\mathbb{P}\left[F_{\mathbf{Y}}(\mathbf{Y}) \geq \alpha\right]}
$$

Note that $X_{i}+Y_{i}$ and $X_{j}+Y_{j}$ are independent for all $i, j=1, \ldots, d$, with $i \neq j$. Then all denominators of the terms above are the same, i.e., the survival Kendall distribution $\bar{K}(\alpha)$ associated to the $d$-dimensional independence copula structure. As a result, we have then to prove that, for all $i=1, \ldots, d$ and for all $\alpha \in(0,1)$

$$
\mathbb{E}\left[X_{i}+Y_{i}, F_{\mathbf{X}}+\mathbf{Y}(\mathbf{X}+\mathbf{Y}) \geq \alpha\right] \leq \mathbb{E}\left[X_{i}, F_{\mathbf{X}}(\mathbf{X}) \geq \alpha\right]+\mathbb{E}\left[Y_{i}, F_{\mathbf{Y}}(\mathbf{Y}) \geq \alpha\right]
$$

Let us denote by $\mathbf{U}=\left(U_{1}, \ldots, U_{d}\right)$ the random vector with components $U_{j}:=F_{X_{j}+Y_{j}}\left(X_{j}+Y_{j}\right), j=$ $1, \ldots, d$ and by $\mathbf{V}=\left(V_{1}, \ldots, V_{d}\right)$ the random vector with components $V_{j}:=F_{X_{j}}\left(X_{j}\right), j=1, \ldots, d$. As the components of $\mathbf{X}+\mathbf{Y}$ are independent, we get:

$$
\mathbb{E}\left[X_{i}+Y_{i}, F_{\mathbf{X}}+\mathbf{Y}(\mathbf{X}+\mathbf{Y}) \geq \alpha\right]=\mathbb{E}\left[X_{i}+Y_{i}, F_{X_{i}+Y_{i}}\left(X_{i}+Y_{i}\right) \geq \tilde{\alpha}_{i}\right]
$$

where 


$$
\tilde{\alpha}:=\frac{\alpha}{\prod_{j=1, j \neq i}^{d} U_{j}} .
$$

Using the sub-additivity of the univariate Conditional-Tail-Expectation (see for instance Denuit et al. (2005)), the following relation holds

$$
\mathbb{E}\left[X_{i}+Y_{i}, F_{X_{i}+Y_{i}}\left(X_{i}+Y_{i}\right) \geq \tilde{\alpha} \mid U_{j, j \neq i}\right] \leq \mathbb{E}\left[X_{i}, F_{X_{i}}\left(X_{i}\right) \geq \tilde{\alpha} \mid U_{j, j \neq i}\right]+\mathbb{E}\left[Y_{i}, F_{Y_{i}}\left(Y_{i}\right) \geq \tilde{\alpha} \mid U_{j, j \neq i}\right] .
$$

Note that the vector $\left(U_{1}, \ldots, X_{i}, \ldots, U_{d}\right)$ has the same distribution as the vector $\left(V_{1}, \ldots, X_{i}, \ldots, V_{d}\right)$ since they both share the same marginals and the same dependence structure (the independent copula). As a result, $\mathbb{E}\left[X_{i}, F_{X_{i}}\left(X_{i}\right) \geq \tilde{\alpha} \mid U_{j, j \neq i}\right]=\mathbb{E}\left[X_{i}, F_{\mathbf{X}}(\mathbf{X}) \geq \alpha \mid V_{j, j \neq i}\right]$. Using the same argument for the second term in the right hand side of (13), the result follows from the law of iterated expectation.

Remark 4. Note that the previous subadditivity property does not hold in general. Lee and Prékopa (2012) provide a counterexample in the case where the underlying random vectors follow particular discrete distributions.

\subsection{Archimedean copula case}

In this section, we focus on multivariate random vectors distributed as particular Archimedean copulas. Note that, since Archimedean copulas are exchangeable, all components of the introduced multivariate risk measures are the same, when applied to random vectors with such distributions. We show that analytical expressions can be obtained for the lower-orthant and the upper-orthant CTE, which allows us to illustrate the behavior of the proposed risk-measures. In the next sections, the presented properties will be investigated more formerly.

We restrain ourself to random vectors with uniform marginals. In that case, the multivariate VaR-s introduced in Cousin and Di Bernardino (2013) can be expressed as an integral transformation of the Archimedean generator.

Definition 2.3. A d-dimensional Archimedean copula $C$ with generator $\phi$ is a distribution function on $(0,1)^{d}$ defined by

$$
C\left(u_{1}, \ldots, u_{d}\right)=\phi^{-1}\left(\phi\left(u_{1}\right)+\cdots+\phi\left(u_{d}\right)\right),
$$

where $\phi^{-1}$ is a d-monotone function from $[0, \infty)$ to $[0,1]$.

Proposition 2.5. Let $\mathbf{X}$ be a d-dimensional random vector which follows an Archimedean copula with generator $\phi$, then, for any $i=1, \ldots, d$,

$$
\begin{aligned}
\underline{\operatorname{VaR}}_{\alpha}^{i}(\mathbf{X}) & =1-\int_{\alpha}^{1}\left(1-\frac{\phi(u)}{\phi(\alpha)}\right)^{d-1} \mathrm{~d} u, \\
\overline{\operatorname{VaR}}_{\alpha}^{i}(\mathbf{1}-\mathbf{X}) & =\int_{1-\alpha}^{1}\left(1-\frac{\phi(u)}{\phi(1-\alpha)}\right)^{d-1} \mathrm{~d} u .
\end{aligned}
$$

Proof: This comes down from Corollary 8 in Cousin and Di Bernardino (2013).

Consequently, by using representations (8)-(9) and relations (14)-(15), the following integral expressions hold for any $i=1, \ldots, d$

$$
\begin{aligned}
\mathrm{CTE}_{\alpha}^{i}(\mathbf{X}) & =\frac{1}{1-K(\alpha)} \int_{\alpha}^{1}\left(1-\int_{\gamma}^{1}\left(1-\frac{\phi(u)}{\phi(\gamma)}\right)^{d-1} \mathrm{~d} u\right) K^{\prime}(\gamma) d \gamma \\
\overline{\operatorname{CTE}}_{\alpha}^{i}(\mathbf{1}-\mathbf{X}) & =\frac{1}{K(1-\alpha)} \int_{\alpha}^{1}\left(\int_{1-\gamma}^{1}\left(1-\frac{\phi(u)}{\phi(1-\gamma)}\right)^{d-1} \mathrm{~d} u\right) K^{\prime}(1-\gamma) d \gamma
\end{aligned}
$$


where $K$ is the Kendall distribution function associated with $X$. Using (16), we are able to give analytical expressions of $\mathrm{CTE}_{\alpha}(\mathbf{X})$ for some particular families of Archimedean copulas. Additionally, from Example 1 , analogous expressions can be obtained for the upper-orthant CTE of the vector $\mathbf{1}-\mathbf{X}=\left(1-X_{1}, \ldots, 1-\right.$ $X_{d}$ ) given that the Kendall distribution $K$ of $\mathbf{X}$ is known. One can use either formula (17) or the following relation

$$
\overline{\mathrm{CTE}}_{\alpha}^{i}(\mathbf{1}-\mathbf{X})=\frac{1}{K(1-\alpha)}\left[\frac{1}{2}-(1-K(1-\alpha))\left(1-\underline{\mathrm{CTE}}_{1-\alpha}^{i}(\mathbf{X})\right)\right]
$$

which comes downs from Equation (10). In Table 1, we provide analytical expressions of the Kendall distribution function $K(\alpha)$ for bivariate Gumbel, Frank, Clayton and Ali-Mikhail-Haq families.

\begin{tabular}{|c|c|c|}
\hline Copula & $\theta \in$ & Kendall distribution $K(\alpha, \theta)$ \\
\hline Gumbel & {$[1, \infty)$} & $\alpha\left(1-\frac{1}{\theta} \ln \alpha\right)$ \\
Frank & $(-\infty, \infty) \backslash\{0\}$ & $\alpha+\frac{1}{\theta}\left(1-\mathrm{e}^{\theta \alpha}\right) \ln \left(\frac{1-\mathrm{e}^{-\theta \alpha}}{1-\mathrm{e}^{-\theta}}\right)$ \\
Clayton & {$[-1, \infty) \backslash\{0\}$} & $\alpha\left(1+\frac{1}{\theta}\left(1-\alpha^{\theta}\right)\right)$ \\
Ali-Mikhail-Haq & {$[-1,1)$} & $\frac{\alpha-1+\theta+(1-\theta+\theta \alpha)(\ln (1-\theta+\theta \alpha)+\ln \alpha)}{\theta-1}$ \\
\hline
\end{tabular}

Table 1: Kendall distribution in some classical bivariate Archimedean copulas.

As a matter of example, we now focus on Clayton and Gumbel copula families.

\section{Clayton family illustration}

Let us now consider the Clayton family of bivariate copulas. This family is interesting since it contains the counter-monotonic, the independence and the comonotonic copulas as particular cases. Clayton copulas are associated with generator $\phi$ of the form $\phi(u)=\frac{1}{\theta}\left(u^{-\theta}-1\right), u \in(0,1)$ with a dependence parameter $\theta$. Let $(X, Y)$ be a random vector distributed as a Clayton copula with parameter $\theta \geq-1$. Then, $X$ and $Y$ are uniformly-distributed on $(0,1)$ and the joint distribution function $C_{\theta}$ of $(X, Y)$ is such that

$$
C_{\theta}(x, y)=\left(\max \left\{x^{-\theta}+y^{-\theta}-1,0\right\}\right)^{-\frac{1}{\theta}}, \quad \text { for } \theta \geq-1, \quad(x, y) \in[0,1]^{2} .
$$

We obtain in Table 2 a closed-form expression for the multivariate lower-orthant $\underline{\mathrm{CTE}}$ in that case.

\begin{tabular}{|c|c|c|}
\hline Copula & $\theta$ & $\underline{\mathrm{CTE}}_{\alpha, \theta}^{i}(X, Y)$ \\
\hline Clayton $C_{\theta}$ & $(-1, \infty)$ & $\frac{1}{2} \frac{\theta}{\theta-1} \frac{\theta-1-\alpha^{2}(1+\theta)+2 \alpha^{1+\theta}}{\theta-\alpha(1+\theta)+\alpha^{1+\theta}}$ \\
Counter-monotonic $W$ & -1 & $\frac{1}{4} \frac{1-\alpha^{2}+2 \ln \alpha}{1-\alpha+\ln \alpha}$ \\
Independent $\Pi$ & 0 & $\frac{1}{2} \frac{(1-\alpha)^{2}}{1-\alpha+\alpha \ln \alpha}$ \\
$\frac{\Pi}{\Sigma-\Pi}$ & 1 & $\frac{1}{2} \frac{1+\alpha^{2}(2 \ln \alpha-1)}{(1-\alpha)^{2}}$ \\
Comonotonic $M$ & $\infty$ & $\frac{1+\alpha}{2}$ \\
\hline
\end{tabular}

Table 2: $\underline{\operatorname{CTE}}_{\alpha, \theta}^{i}(X, Y), i=1,2$, for different copula dependence structures.

Interestingly, one can readily show that $\frac{\partial \mathrm{CTE}_{\alpha, \theta}^{i}}{\partial \alpha} \geq 0$ and $\frac{\partial \mathrm{CTE}_{\alpha, \theta}^{i}}{\partial \theta} \leq 0$, for $\theta \geq-1$ and $\alpha \in(0,1)$. This proves that, for Clayton-distributed random vectors, the components of our CTE are increasing functions of the risk level $\alpha$ and decreasing functions of the dependence parameter $\theta$. Note also that, in the comonotonic case, our CTE corresponds to the vector composed of the univariate CTE associated with each component. These properties are illustrated in Figure 1 (left panel) where $\underline{\mathrm{CTE}}_{\alpha, \theta}^{i}(X, Y)$ is plotted as a function of the risk level $\alpha$ for different values of $\theta$. Observe that an increase of the dependence parameter $\theta$ tends to lower the $\underline{\mathrm{CTE}}$ up to the perfect dependence case where $\underline{\mathrm{CTE}}_{\alpha, \theta=+\infty}^{i}(X, Y)=\mathrm{CTE}_{\alpha}(X)=\frac{1+\alpha}{2}$. The previous empirical behaviors will be formally investigated in next sections. 
In the same framework, using Equation (18), one can readily show that $\frac{\partial \overline{\mathrm{CTE}}_{\alpha, \theta}^{i}}{\partial \alpha} \geq 0$ and $\frac{\partial \overline{\mathrm{CTE}}_{\alpha, \theta}^{i}}{\partial \theta} \geq 0$, for $\theta \geq-1$ and $\alpha \in(0,1)$. This proves that, for random couples with uniform margins and Clayton survival copula, the components of our multivariate $\overline{\mathrm{CTE}}$ are increasing functions both of the risk level $\alpha$ and of the dependence parameter $\theta$. Note also that the multivariate $\overline{\mathrm{CTE}}$ in the comonotonic case corresponds to the vector composed of the univariate CTE associated with each component. These properties are illustrated in Figure 1 (right panel) where $\overline{\mathrm{CTE}}_{\alpha, \theta}^{i}(1-X, 1-Y)$ is plotted as a function of the risk level $\alpha$ for different values of the parameter $\theta$. Observe that, contrary to the lower-orthant $\underline{\mathrm{CTE}}$, an increase of the dependence parameter $\theta$ tends to increase the $\overline{\mathrm{CTE}}$. Then in the case of upper-orthant CTE, the upper bound is represented by the perfect dependence case where $\overline{\mathrm{CTE}}_{\alpha, \theta=+\infty}^{1}(X, Y)=\mathrm{CTE}_{\alpha}(X)=\frac{1+\alpha}{2}$. The latter empirical behaviors will be formally confirmed in next sections.
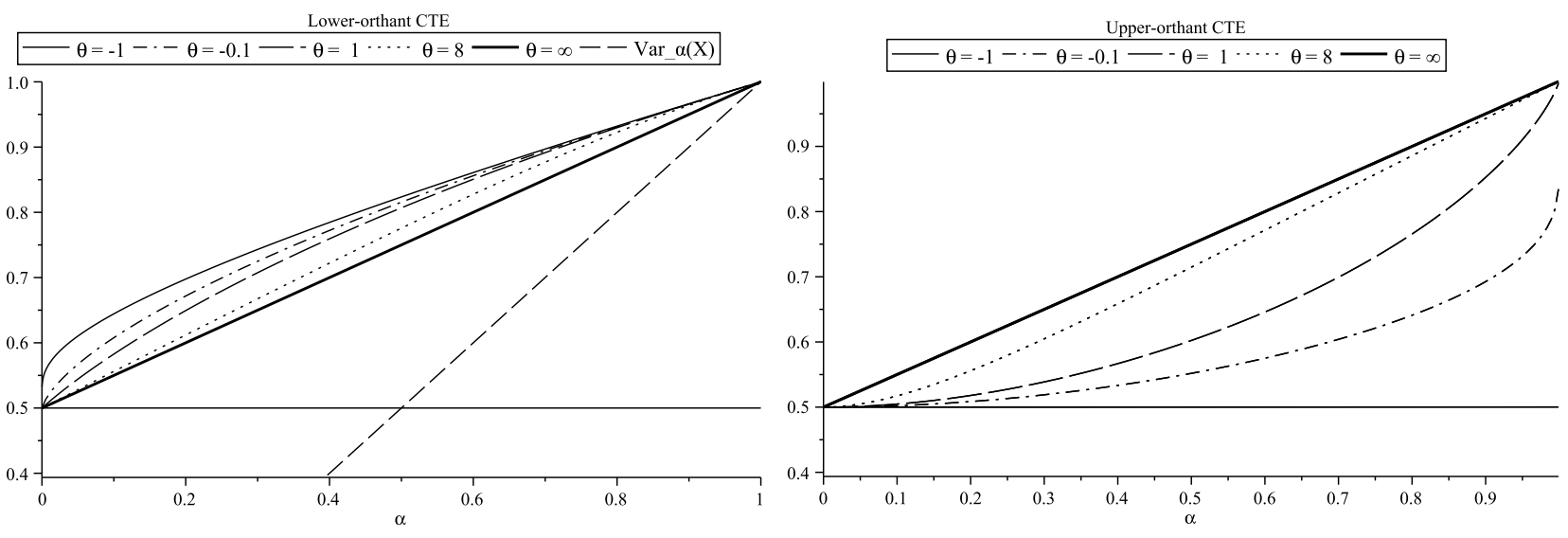

Figure 1: Behavior of $\underline{\mathrm{CTE}}_{\alpha, \theta}^{1}(X, Y)=\underline{\mathrm{CTE}}_{\alpha, \theta}^{2}(X, Y)$ (left) and $\overline{\mathrm{CTE}}_{\alpha, \theta}^{1}(1-X, 1-Y)=\overline{\mathrm{CTE}}_{\alpha, \theta}^{2}(1-X, 1-Y)$ (right) with respect to risk level $\alpha$ for different values of dependence parameter $\theta$. The random vector $(X, Y)$ follows a Clayton copula distribution with parameter $\theta$. The horizontal line corresponds to $\mathbb{E}[X]=\frac{1}{2}$. Note that this limit is reached (right panel) for the upper-orthant CTE in the counter-monotonic case, i.e., $\overline{\mathrm{CTE}}_{\alpha, \theta=-1}^{1}(1-$ $X, 1-Y)=\frac{1}{2}$.

Gumbel family illustration

Gumbel copulas are associated with a generator $\phi$ of the form $\phi(u)=(-\log (u))^{\theta}, u \in(0,1)$ with a dependence parameter $\theta \geq 1$. The bivariate family of copulas is such that

$$
C_{\theta}(x, y)=\mathrm{e}^{-\left((-\ln x)^{\theta}+(-\ln y)^{\theta}\right)^{\frac{1}{\theta}}},
$$

for $\theta \in[1, \infty),(x, y) \in[0,1]^{2}$ (e.g., see Section 3.3.1 in Nelsen (1999)) and $X, Y$ standard uniform marginals. For $\theta=1$ we have the independent copula $C_{1}(x, y)=\Pi(x, y)=x y$; for $\theta=\infty$ the Fréchet bound $M(x, y)=\min \{x, y\}$ (comonotonic random variables). Again, in this case, analytical expressions of $\underline{\mathrm{CTE}}_{\alpha}(X, Y)$ and $\overline{\mathrm{CTE}}_{\alpha, \theta}^{1}(1-X, 1-Y)$ can be derived. As in the Clayton case, it can be proved that $\frac{\partial \mathrm{CTE}_{\alpha, \theta}^{1}}{\partial \alpha} \geq 0, \frac{\partial \mathrm{CTE}_{\alpha, \theta}^{1}}{\partial \theta} \leq 0, \frac{\partial \overline{\mathrm{CTE}}_{\alpha, \theta}^{1}}{\partial \alpha} \geq 0$ and $\frac{\partial \overline{\mathrm{CTE}}_{\alpha, \theta}^{1}}{\partial \theta} \geq 0$, for $\theta \geq 1$ and $\alpha \in(0,1)$. Figure 2 plots one component of the proposed measures in the Gumbel case (all components are the same by exchangeability), as a function of the risk level $\alpha$ and for different dependence parameter $\theta$. We can see that the behavior of the upper-orthant and the lower-orthant CTE are similar to the one exhibited in Figure 1 for the Clayton case.

\subsection{Comparison with other risk measures}

We now discuss whether the components of $\underline{\mathrm{CTE}}$ and $\overline{\mathrm{CTE}}$ can be compared with univariate VaR and CTE applied to the corresponding marginal risks and under which condition components of CTE and $\overline{\mathrm{CTE}}$ are more conservative that the components of $\underline{\mathrm{VaR}}$ and $\overline{\mathrm{VaR}}$. 

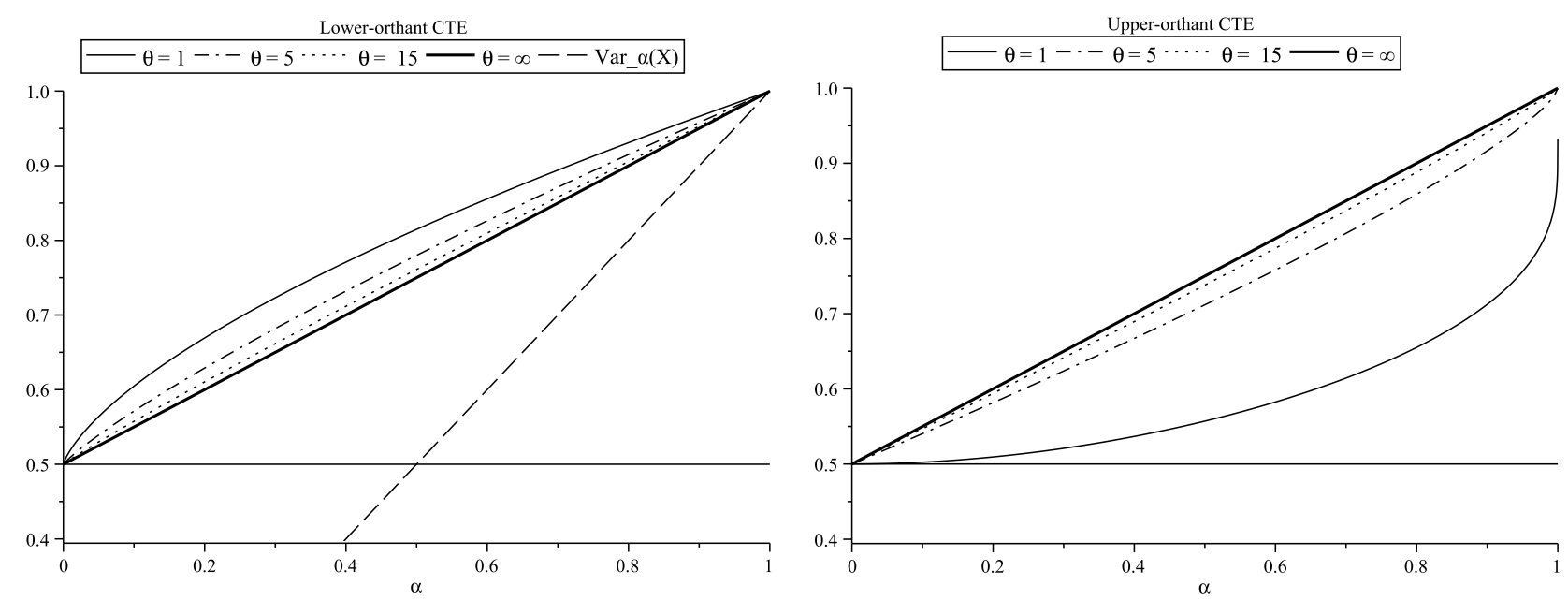

Figure 2: Behavior of $\underline{\mathrm{CTE}}_{\alpha, \theta}^{1}(X, Y)=\underline{\mathrm{CTE}}_{\alpha, \theta}^{2}(X, Y)($ left $)$ and $\overline{\mathrm{CTE}}_{\alpha, \theta}^{1}(1-X, 1-Y)=\overline{\mathrm{CTE}}_{\alpha, \theta}^{2}(1-X, 1-Y)$ (right) with respect to risk level $\alpha$ for different values of dependence parameter $\theta$. The random vector $(X, Y)$ follows a Gumbel copula distribution with parameter $\theta$. The horizontal line corresponds to $\mathbb{E}[X]=\frac{1}{2}$.

Proposition 2.6. Consider a d-dimensional random vector $\boldsymbol{X}$. Assume that its multivariate distribution function $F$ is quasi concave $e^{5}$ Then, for any $i=1, \ldots, d$, the following inequality holds:

$$
\operatorname{VaR}_{\alpha}\left(X_{i}\right) \leq \underline{\operatorname{CTE}}_{\alpha}^{i}(\boldsymbol{X}), \quad \text { for } i=1, \ldots, d \text {. }
$$

Proof: Let $\alpha \in(0,1)$. From the definition of the accumulated probability, it is easy to show that $\partial \underline{L}(\alpha)$ is inferiorly bounded by the marginal univariate quantile functions. Moreover, recall that $\underline{L}(\alpha)$ is a convex set in $\mathbb{R}_{+}^{d}$ from the quasi concavity of $F$ (see Section 2 in Tibiletti (1995)). Then, for all $\mathbf{x}=\left(x_{1}, \ldots, x_{d}\right) \in \underline{L}(\alpha), x_{1} \geq \operatorname{VaR}_{\alpha}\left(X_{1}\right), \cdots, x_{d} \geq \operatorname{VaR}_{\alpha}\left(X_{d}\right)$ and trivially, $\underline{C T E}_{\alpha}^{i}(\mathbf{X})$ is greater than $\operatorname{VaR}_{\alpha}\left(X_{i}\right)$, for $i=1, \ldots, d$. Hence the result.

Proposition 2.6 states that the multivariate lower-orthant $\underline{\mathrm{CTE}}_{\alpha}$ is more conservative than the vector composed of the classical univariate $\alpha$-Value-at-Risk of marginal distributions. Such a result is not true for the upper-orthant CTE as can be seen in left panel of Figures 1-2.

It is interesting to remark that, for comonotonic random vectors, the proposed multivariate CTE-s coincide with the vector composed of univariate CTE-s of the corresponding marginals.

Proposition 2.7. Consider a comonotonic non-negative d-dimensional random vector $\boldsymbol{X}$. Then, for all $\alpha \in(0,1)$, it holds that

$$
\underline{\operatorname{CTE}}_{\alpha}^{i}(\boldsymbol{X})=\operatorname{CTE}_{\alpha}\left(X_{i}\right)=\overline{\operatorname{CTE}}_{\alpha}^{i}(\boldsymbol{X}), \quad \text { for } i=1, \ldots, d \text {. }
$$

Proof: If $\mathbf{X}=\left(X_{1}, \ldots, X_{d}\right)$ is a comonotonic non-negative random vector then there exist a random variable $Z$ and $d$ increasing functions $g_{1}, \ldots, g_{d}$ such that $\mathbf{X}$ is equal to $\left(g_{1}(Z), \ldots, g_{d}(Z)\right)$ in distribution. So $F_{X}(x)=F_{Z}\left(\min _{i=1, \ldots, d}\left\{g_{i}^{-1}\left(x_{i}\right)\right\}\right)$ and $\bar{F}_{X}(x)=\bar{F}_{Z}\left(\max _{i=1, \ldots, d}\left\{g_{i}^{-1}\left(x_{i}\right)\right\}\right)$ so that $F_{X}(X)=F_{Z}(Z)=$ $F_{X_{i}}\left(X_{i}\right)$ and $\bar{F}_{X}(X)=\bar{F}_{Z}(Z)=\bar{F}_{X_{i}}\left(X_{i}\right)$ for all $i=1, \ldots, d$. The result follows immediately.

Let us now compare the multivariate lower-orthant and upper-orthant CTE introduced in Definition 2.1 with the multivariate lower-orthant and upper-orthant VaR defined by Cousin and Di Bernardino (2013).

\footnotetext{
${ }^{5}$ A function $F$ is quasi concave if for any $x, y, F(p x+(1-p) y) \geq \min (F(x), F(y))$ for all $p \in(0,1)$. Note that a function $F$ is quasi concave if and only if its upper level sets of are convex. Tibiletti (1995) points out families of distribution functions which satisfy the property of quasi concavity. For instance, multivariate elliptical distributions and Archimedean copulas are quasi concave functions.
} 
Proposition 2.8. Consider a d-dimensional random vector $\boldsymbol{X}$ satisfying the regularity conditions. If $\underline{\operatorname{VaR}}_{\alpha}^{i}(\boldsymbol{X})$ is a non-decreasing function of $\alpha$, then it holds that

$$
\underline{\operatorname{CTE}}_{\alpha}^{i}(\boldsymbol{X}) \geq \underline{\operatorname{VaR}}_{\alpha}^{i}(\boldsymbol{X}), \quad \text { for all } \alpha \in(0,1) \text {. }
$$

If $\overline{\operatorname{VaR}}_{\alpha}^{i}(\boldsymbol{X})$ is a non-decreasing function of $\alpha$, then it holds that

$$
\overline{\mathrm{CTE}}_{\alpha}^{i}(\boldsymbol{X}) \geq \overline{\operatorname{VaR}}_{\alpha}^{i}(\boldsymbol{X}), \quad \text { for all } \alpha \in(0,1) .
$$

Proof:

From Equation (8), since $\underline{\operatorname{VaR}}_{\gamma}^{i}(\mathbf{X})$ is assumed to be non-decreasing with respect to $\gamma$, we obtain

$$
\underline{\operatorname{CTE}}_{\alpha}^{i}(\mathbf{X}) \geq \frac{1}{1-K(\alpha)} \int_{\alpha}^{1} \underline{\operatorname{VaR}}_{\alpha}^{i}(\mathbf{X}) K^{\prime}(\gamma) \mathrm{d} \gamma=\frac{K(1)-K(\alpha)}{1-K(\alpha)} \underline{\operatorname{VaR}}_{\alpha}^{i}(\mathbf{X})=\underline{\operatorname{VaR}}_{\alpha}^{i}(\mathbf{X}) .
$$

From Equation (9), if $\overline{\operatorname{VaR}}_{\gamma}^{i}(\mathbf{X})$ is assumed to be non-decreasing with respect to $\gamma$, we obtain

$$
\overline{\mathrm{CTE}}_{\alpha}^{i}(\mathbf{X}) \geq \frac{1}{\widehat{K}(1-\alpha)} \int_{\alpha}^{1} \overline{\operatorname{VaR}}_{\alpha}^{i}(\mathbf{X}) \widehat{K}^{\prime}(1-\gamma) \mathrm{d} \gamma=\frac{\widehat{K}(1-\alpha)-\widehat{K}(0)}{\widehat{K}(1-\alpha)} \overline{\operatorname{VaR}}_{\alpha}^{i}(\mathbf{X})=\overline{\operatorname{VaR}}_{\alpha}^{i}(\mathbf{X}) .
$$

Hence the result.

This property holds for instance for any random vector which admits an Archimedean copula. It is indeed proved in Cousin and Di Bernardino (2013) that $\underline{\operatorname{VaR}}_{\alpha}(\mathbf{X})$ and $\overline{\mathrm{VaR}}_{\alpha}(\mathbf{X})$ are non-decreasing functions of the risk level $\alpha$ for such dependence structures.

\subsection{Behavior of multivariate CTE with respect to marginal distributions}

In this section we study the behavior of the multivariate lower-orthant and upper-orthant ConditionalTail-Expectation with respect to a change in marginal distributions. Results presented below provide a natural multivariate extension of classical results in a univariate setting (see, e.g., Denuit and Charpentier (2004)).

Proposition 2.9. Let $\boldsymbol{X}$ and $\boldsymbol{Y}$ be two d-dimensional random vector satisfying the regularity conditions and with the same copula structure $C$. If $X_{i} \stackrel{d}{=} Y_{i}$, then it holds that

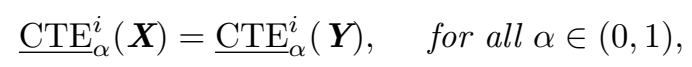

and

$$
\overline{\mathrm{CTE}}_{\alpha}^{i}(\boldsymbol{X})=\overline{\mathrm{CTE}}_{\alpha}^{i}(\boldsymbol{Y}), \quad \text { for all } \alpha \in(0,1) .
$$

Proof: From Proposition 2.6 in Cousin and Di Bernardino (2013), we know that $\underline{\operatorname{VaR}}_{\alpha}^{i}(\mathbf{X})=\underline{\operatorname{VaR}}_{\alpha}^{i}(\mathbf{Y})$ and $\overline{\operatorname{VaR}}_{\alpha}^{i}(\mathbf{X})=\overline{\operatorname{VaR}}_{\alpha}^{i}(\mathbf{Y})$. The demonstration comes down from representation (8) and (9).

In particular, when one component of the underlying risk vector changes, it does not affect the other components of the multivariate CTE-s as far as the dependence structure is unchanged. In the following, we analyze how our multivariate measures behave when the marginal risks increase with respect to the first-order stochastic dominance.

Proposition 2.10. Let $\boldsymbol{X}$ and $\boldsymbol{Y}$ be two d-dimensional random vectors satisfying the regularity conditions and with the same copula structure $C$. If $X_{i} \preceq_{s t} Y_{i}$ then it holds that

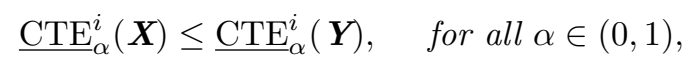

and

$$
\overline{\mathrm{CTE}}_{\alpha}^{i}(\boldsymbol{X}) \leq \overline{\mathrm{CTE}}_{\alpha}^{i}(\boldsymbol{Y}), \quad \text { for all } \alpha \in(0,1) .
$$

Proof: From Proposition 2.7 in Cousin and Di Bernardino (2013), we know that $\operatorname{VaR}_{\alpha}^{i}(\mathbf{X}) \leq \operatorname{VaR}_{\alpha}^{i}(\mathbf{Y})$ and $\overline{\operatorname{VaR}}_{\alpha}^{i}(\mathbf{X}) \leq \overline{\operatorname{VaR}}_{\alpha}^{i}(\mathbf{Y})$. The demonstration comes down from representation (8) and (9). 
Example 2. In this example, we provide an illustration of Propositions 2.9-2.10 above. We consider the case of lower-orthant Conditional-Tail-Expectation (the upper-orthant case is completly analogous). The obtained results are gathered in Table 3.

We consider five different bivariate random vectors $\left(X, Y_{i}\right)$, for $i=1, \ldots, 5$, with the same bivariate Clayton copula with parameter 1 . Let

$$
X \sim \operatorname{Exp}(1), Y_{1} \sim \operatorname{Exp}(2), Y_{2} \sim \operatorname{Burr}(2,1), Y_{3} \sim \operatorname{Exp}(1), Y_{4} \sim \text { Fréchet(4) and } Y_{5} \sim \operatorname{Burr}(4,1) .
$$

We calculate $\underline{\mathrm{CTE}}_{\alpha}\left(X, Y_{i}\right)$, for $i=1, \ldots, 5$ (see Table 3). As proved in Proposition 2.9 we obtain an invariant property on the first coordinate of all $\underline{\mathrm{CTE}}_{\alpha}\left(X, Y_{i}\right)$, for $i=1, \ldots, 5$. Furthermore, since $\left(X, Y_{3}\right)$ is an exchangeable continuous random vector, then $\underline{\operatorname{CTE}}_{\alpha}^{1}\left(X, Y_{3}\right)=\underline{\operatorname{CTE}}_{\alpha}^{2}\left(X, Y_{3}\right)$. Moreover, as $Q_{X}(\alpha)=2 Q_{Y_{1}}(\alpha)$, then $\underline{\mathrm{CTE}}_{\alpha}^{1}\left(X, Y_{1}\right)=2 \underline{\mathrm{CTE}}_{\alpha}^{2}\left(X, Y_{1}\right)$, for $\alpha \in(0,1)$ (see Table 3$)$.

By in Proposition 2.10, since $Y_{1} \preceq_{s t} Y_{5} \preceq_{s t} Y_{4} \preceq_{s t} Y_{2}$, then,

$$
\underline{\mathrm{CTE}}_{\alpha}^{2}\left(X, Y_{1}\right) \leq \underline{\mathrm{CTE}_{\alpha}^{2}}\left(X, Y_{5}\right) \leq \underline{\mathrm{CTE}_{\alpha}^{2}}\left(X, Y_{4}\right) \leq \underline{\mathrm{CTE}_{\alpha}^{2}}\left(X, Y_{2}\right) \text {, for any level } \alpha \in(0,1) .
$$

Analogously, we also obtain $\underline{\mathrm{CTE}}_{\alpha}\left(X, Y_{1}\right) \leq \underline{\mathrm{CTE}}_{\alpha}\left(X, Y_{3}\right) \leq \underline{\mathrm{CTE}}_{\alpha}\left(X, Y_{2}\right)$, for any level $\alpha \in(0,1)$. Conversely $Y_{3}, Y_{4}$ and $Y_{3}, Y_{5}$ are not ordered in stochastic dominance sense.

\begin{tabular}{|c|c|c|c|c|c|}
\hline$\alpha$ & $\mathrm{CTE}_{\alpha}\left(X, Y_{1}\right)$ & $\mathrm{CTE}_{\alpha}\left(X, Y_{2}\right)$ & $\underline{\mathrm{CTE}}_{\alpha}\left(X, Y_{3}\right)$ & $\underline{\mathrm{CTE}}_{\alpha}\left(X, Y_{4}\right)$ & $\underline{\mathrm{CTE}_{\alpha}\left(X, Y_{5}\right)}$ \\
\hline 0.10 & $(1.188,0.594)$ & $(1.188,1.838)$ & $(1.188,1.188)$ & $(1.188,1.315)$ & $(1.188,1.229)$ \\
0.24 & $(1.449,0.724)$ & $(1.449,2.218)$ & $(1.449,1.449)$ & $(1.449,1.431)$ & $(1.449,1.366)$ \\
0.38 & $(1.727,0.864)$ & $(1.727,2.661)$ & $(1.727,1.727)$ & $(1.727,1.555)$ & $(1.727,1.506)$ \\
0.52 & $(2.049,1.025)$ & $(2.049,3.235)$ & $(2.049,2.049)$ & $(2.049,1.704)$ & $(2.049,1.667)$ \\
0.66 & $(2.454,1.227)$ & $(2.454,4.074)$ & $(2.454,2.454)$ & $(2.454,1.902)$ & $(2.454,1.876)$ \\
0.80 & $(3.039,1.519)$ & $(3.039,5.591)$ & $(3.039,3.039)$ & $(3.039,2.219)$ & $(3.039,2.202)$ \\
0.90 & $(3.768,1.884)$ & $(3.768,8.175)$ & $(3.768,3.768)$ & $(3.768,2.675)$ & $(3.768,2.665)$ \\
0.99 & $(6.102,3.059)$ & $(6.102,26.59)$ & $(6.102,6.102)$ & $(6.102,4.813)$ & $(6.102,4.811)$ \\
\hline
\end{tabular}

Table 3: $\underline{\mathrm{CTE}}_{\alpha}\left(X, Y_{i}\right)$, for $i=1, \ldots, 5$, with the same copula Clayton copula with parameter 1, $X \sim \operatorname{Exp}(1)$ and $Y_{1} \sim \operatorname{Exp}(2) ; Y_{2} \sim \operatorname{Burr}(2,1) ; Y_{3} \sim \operatorname{Exp}(1) ; Y_{4} \sim \operatorname{Fréchet}(4) ; Y_{5} \sim \operatorname{Burr}(4,1)$. This table provides an illustration of Propositions 2.9-2.10.

In the following proposition, we investigate the effect of a mean-preserving change in marginal risks.

Proposition 2.11. Let $\boldsymbol{X}$ and $\boldsymbol{Y}$ be two d-dimensional random vectors satisfying the regularity conditions and with the same copula structure $C$. Let us assume that $\mathbb{E}\left[X_{i}\right] \leq \mathbb{E}\left[Y_{i}\right]$ and that the ith components of the lower-orthant $V a R$ of $X$ and $Y$ satisfy the following single-cut condition: there exists a real $c$ in $[0,1)$ such that $\underline{\operatorname{VaR}}_{\alpha}^{i}(\boldsymbol{X}) \geq \underline{\operatorname{VaR}}_{\alpha}^{i}(\boldsymbol{Y})$ for all $\alpha<c$ and $\underline{\operatorname{VaR}}_{\alpha}^{i}(\boldsymbol{X}) \leq \underline{\operatorname{VaR}}_{\alpha}^{i}(\boldsymbol{Y})$ for all $\alpha \geq$. Then,

$$
\underline{\mathrm{CTE}}_{\alpha}^{i}(\boldsymbol{X}) \leq \underline{\mathrm{CTE}}_{\alpha}^{i}(\boldsymbol{Y}), \quad \text { for all } \alpha \in(0,1) .
$$

The same result holds $\overline{\mathrm{CTE}}$.

Proof: Let us consider the function $\Delta$ defined by

$$
\begin{aligned}
\alpha \mapsto \Delta(\alpha) & =(1-K(\alpha))\left(\underline{\mathrm{CTE}}_{\alpha}^{i}(\mathbf{Y})-\underline{\mathrm{CTE}}_{\alpha}^{i}(\mathbf{X})\right) \\
& =\int_{\alpha}^{1}\left(\underline{\operatorname{VaR}}_{\gamma}^{i}(\mathbf{Y})-\underline{\operatorname{VaR}}_{\gamma}^{i}(\mathbf{X})\right) K^{\prime}(\gamma) \mathrm{d} \gamma
\end{aligned}
$$

Then $\Delta(0)=(1-K(0))\left(\mathbb{E}\left[Y_{i}\right]-\mathbb{E}\left[X_{i}\right]\right) \geq 0, \Delta(1)=0$ and $\Delta^{\prime}(\alpha)=\left(\underline{\operatorname{VaR}}_{\alpha}^{i}(\mathbf{X})-\underline{\operatorname{VaR}}_{\alpha}^{i}(\mathbf{Y})\right) K^{\prime}(\alpha)$ is positive for all $\alpha<c$ and negative for all $\alpha \geq c$ thanks to the single-cut condition. As a result, the function $\Delta$ remains positive on $[0,1]$ and the result holds. 
This result is similar to the consistency property of univariate CTE with respect to the less dangerous order, i.e., for univariate random variables $X$ and $Y$ such as $X \preceq_{D} Y$, then, for any $\alpha$ in $[0,1]$, $\mathrm{CTE}_{\alpha}(X) \leq \operatorname{CTE}_{\alpha}(Y)$

Example 3. We provide here an illustration of Proposition 2.11. Firstly we remark in Example 2 that $\mathbb{E}\left[Y_{3}\right]<\mathbb{E}\left[Y_{5}\right]$. However, the single-cut condition of Proposition 2.11 for $\underline{\operatorname{VaR}}_{\alpha}^{2}\left(X, Y_{3}\right)$ and $\underline{\operatorname{VaR}}_{\alpha}^{2}\left(X, Y_{5}\right)$ is not satisfied and from Table 3 one can see that $\underline{\mathrm{CTE}}_{\alpha}^{2}\left(X, Y_{3}\right), \underline{\mathrm{CTE}}_{\alpha}^{2}\left(X, Y_{5}\right)$ are not ordered for any level $\alpha \in(0,1)$.

We now define $\mathbf{X}:=\left(X, Z_{1}\right)$ and $\mathbf{Y}:=\left(X, \frac{1}{2}\left(Z_{1}+Z_{2}\right)\right)$. Let $X$ a continuous random variable. Let $Z_{1}$, $Z_{2}$ be two independent random variables such that $Z_{1} \sim Z_{2} \sim \operatorname{Exp}(2)$, then $Z_{1}+Z_{2} \sim \operatorname{Erlang}(2,2)$ distribution. Assume that $\mathbf{X}$ and $\mathbf{Y}$ have both the same independent copula $C$. One can show that $\mathbb{E}\left[\frac{1}{2}\left(Z_{1}+Z_{2}\right)\right]=\mathbb{E}\left[Z_{1}\right]=\frac{1}{2}$ and there exists a real $c$ in $[0,1)$ such that $\underline{\operatorname{VaR}}_{\alpha}^{2}(\mathbf{Y}) \geq \underline{\operatorname{VaR}}_{\alpha}^{2}(\mathbf{X})$ for all $\alpha<c$ and $\underline{\operatorname{VaR}}_{\alpha}^{2}(\mathbf{Y}) \leq \underline{\operatorname{VaR}}_{\alpha}^{2}(\mathbf{X})$ for all $\alpha \geq c$ (see Figure 3, left). The interested reader is also referred to Section 7.3 in Denuit et al. (2005).

Then, using Proposition (2.11) we obtain $\underline{\mathrm{CTE}}_{\alpha}^{2}(\mathbf{Y}) \leq \underline{\mathrm{CTE}}_{\alpha}^{2}(\mathbf{X})$, for all $\alpha \in(0,1)$. This property is illustrated in Figure 3 (right).
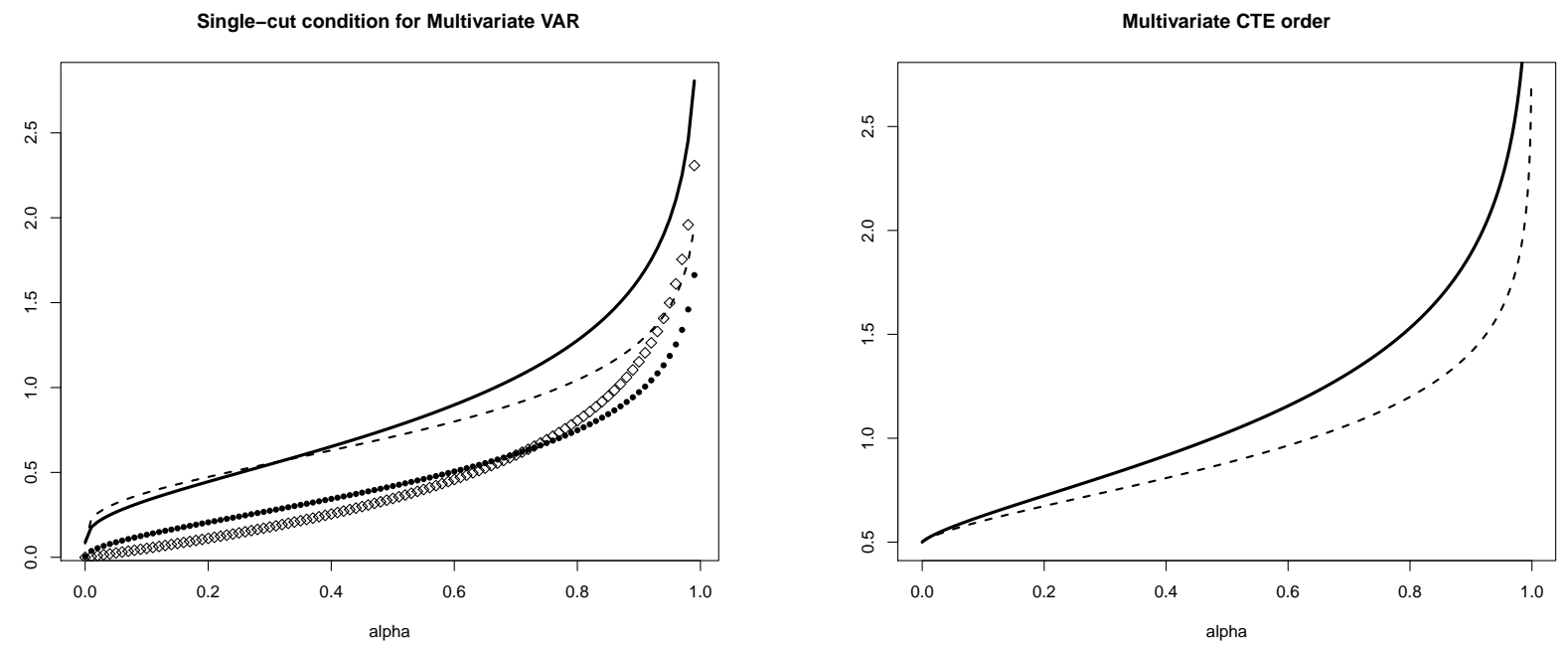

Figure 3: Left: $\underline{\operatorname{VaR}}_{\alpha}^{2}(\mathbf{X})$ (full line) and $\underline{\operatorname{VaR}}_{\alpha}^{2}(\mathbf{Y})$ (dashed line). We also represent the associated univariate $\operatorname{VaR}$, i.e. $\operatorname{VaR}_{\alpha}\left(Z_{1}\right)$ (squared line) and $\operatorname{VaR}_{\alpha}\left(\frac{1}{2}\left(Z_{1}+Z_{2}\right)\right.$ ) (dotted line). Remark that $\underline{\operatorname{VaR}}_{\alpha}^{2}(\mathbf{X})$ and $\underline{\operatorname{VaR}}_{\alpha}^{2}(\mathbf{Y})$ verify the single-cut condition in Proposition 2.11. Right: $\underline{\mathrm{CTE}}_{\alpha}^{2}(\mathbf{X})$ (full line) and $\underline{\mathrm{CTE}} \underline{\mathrm{C}}_{\alpha}^{2}(\mathbf{Y})$ (dashed line).

\subsection{Behavior of multivariate CTE with respect to the dependence structure}

In this section we study the behavior of our CTE generalizations with respect to a variation of the dependence structure, with unchanged marginal distributions.

Proposition 2.12. Let $\boldsymbol{X}$ and $\boldsymbol{X}^{*}$ be two d-dimensional continuous random vectors satisfying the regularity conditions and with the same margins $F_{X_{i}}$ and $F_{X_{i}^{*}}$, for $i=1, \ldots, d$, and let $C$ (resp. $\left.C^{*}\right)$ denote the copula function associated with $\boldsymbol{X}\left(\right.$ resp. $\left.\boldsymbol{X}^{*}\right)$ and $\bar{C}\left(\right.$ resp. $\left.\bar{C}^{*}\right)$ the survival copula function associated with $\boldsymbol{X}$ (resp. $\left.\boldsymbol{X}^{*}\right)$.

Let $U_{i}=F_{X_{i}}\left(X_{i}\right), U_{i}^{*}=F_{X_{i}}{ }\left(X_{i}^{*}\right), \boldsymbol{U}=\left(U_{1}, \ldots, U_{d}\right)$ and $\boldsymbol{U}^{*}=\left(U_{1}^{*}, \ldots, U_{d}^{*}\right)$.

$$
\text { If }\left[U_{i} \mid C(\boldsymbol{U}) \geq \alpha\right] \preceq_{s t}\left[U_{i}^{*} \mid C^{*}\left(\boldsymbol{U}^{*}\right) \geq \alpha\right] \text { then } \underline{\mathrm{CTE}}_{\alpha}^{i}(\boldsymbol{X}) \leq \underline{\operatorname{CTE}}_{\alpha}^{i}\left(\boldsymbol{X}^{*}\right) \text {. }
$$

Let $V_{i}=\bar{F}_{X_{i}}\left(X_{i}\right), V_{i}^{*}=\bar{F}_{X_{i}}\left(X_{i}^{*}\right), \boldsymbol{V}=\left(V_{1}, \ldots, V_{d}\right)$ and $\boldsymbol{V}^{*}=\left(V_{1}^{*}, \ldots, V_{d}^{*}\right)$. 


$$
\text { If }\left[V_{i} \mid \bar{C}(\boldsymbol{V}) \leq 1-\alpha\right] \preceq_{s t}\left[V_{i}^{*} \mid \bar{C}^{*}\left(\boldsymbol{V}^{*}\right) \leq 1-\alpha\right] \text { then } \overline{\mathrm{CTE}}_{\alpha}^{i}(\boldsymbol{X}) \geq \overline{\operatorname{CTE}}_{\alpha}^{i}\left(\boldsymbol{X}^{*}\right) .
$$

Proof: We recall that $U_{1} \preceq_{s t} U_{2}$ if and only if $\mathbb{E}\left[f\left(U_{1}\right)\right] \leq \mathbb{E}\left[f\left(U_{2}\right)\right]$, for all non-decreasing function $f$, such that the expectations exist (see Denuit et al. (2005); Proposition 3.3.14). We now choose $f(u)=Q_{X_{i}}(u)$, for $u \in(0,1)$. Then we obtain

$$
\mathbb{E}\left[Q_{X_{i}}\left(U_{i}\right) \mid C(\mathbf{U}) \geq \alpha\right] \leq \mathbb{E}\left[Q_{X_{i}}\left(U_{i}^{*}\right) \mid C^{*}\left(\mathbf{U}^{*}\right) \geq \alpha\right],
$$

But the right-hand side of the previous inequality is equal to $\mathbb{E}\left[Q_{X_{i}^{*}}\left(U_{i}^{*}\right) \mid C^{*}\left(\mathbf{U}^{*}\right) \geq \alpha\right]$ since $X_{i}$ and $X_{i}^{*}$ have the same distribution. Finally, we obtain $\underline{\mathrm{CTE}}_{\alpha}^{i}(\mathbf{X}) \leq \underline{\mathrm{CTE}}_{\alpha}^{i}\left(\mathbf{X}^{*}\right)$. For the second point of the statement we choose the non-decreasing function $f(u)=-\bar{F}_{X_{i}}^{-1}(u)$, for $u \in(0,1)$. Since $X_{i}$ and $X_{i}^{*}$ have the same distribution, we obtain the result.

We now provide an illustration of Proposition 2.12 in the case of bivariate Archimedean copulas.

Corollary 2.2. Consider a 2-dimensional random vector $\boldsymbol{X}$, satisfying the regularity conditions, with marginal distributions $F_{X_{i}}$, for $i=1, \ldots, d$, copula $C$ and survival copula $\bar{C}$.

If $C$ belongs to one of the bivariate family of Archimedean copulas introduced in Table 1, an increase of the dependence parameter $\theta$ yields a decrease in each component of $\underline{\mathrm{CTE}}_{\alpha}(\boldsymbol{X})$.

If $\bar{C}$ belongs to one of the d-dimensional family of Archimedean copulas introduced in Table 1, an increase of the dependence parameter $\theta$ yields an increase in each component of $\overline{\mathrm{CTE}}_{\alpha}(\boldsymbol{X})$.

Proof: In the bivariate Archimedean case, the joint distribution of $(U, C(U, V))$ can be obtained analytically by using a change of variable transformation ${ }^{6}$ from $(U, V)$ to $(U, C(U, V))$ :

$$
F_{(U, C(U, V))}(u, \alpha)=\alpha-\frac{\phi(\alpha)}{\phi^{\prime}(\alpha)}+\frac{\phi(u)}{\phi^{\prime}(\alpha)}, \quad 0<\alpha<u<1 .
$$

Then, thanks to formula (21), we can obtain the joint survival probability

$$
h(u, \alpha):=\mathbb{P}[U \geq u, C(U, V) \geq \alpha]=1-u+\frac{\phi(u)}{\phi^{\prime}(\alpha)} \quad \text { for } \quad 0<\alpha<u<1 .
$$

Note that $1-K(\alpha)=\mathbb{P}[C(U, V) \geq \alpha]=h(\alpha, \alpha)$. Let $C_{\theta}$ and $C_{\theta^{*}}$ be two bivariate Archimedean copulas of the same family with generator $\phi_{\theta}$ and $\phi_{\theta^{*}}$ such that $\theta \leq \theta^{*}$. Given Proposition 2.12 and by exchangeability, we only have to check that the relation $\left[U^{*} \mid C_{\theta^{*}}\left(\bar{U}^{*}, V^{*}\right) \geq \alpha\right] \preceq_{s t}\left[U \mid C_{\theta}(U, V) \geq \alpha\right]$ hold where $(U, V)$ and $\left(U^{*}, V^{*}\right)$ are distributed according to (resp.) $C_{\theta}$ and $C_{\theta^{*}}$. Given formula (22), the previous relation can be restated as

$$
\frac{h^{*}(u, \alpha)}{h^{*}(\alpha, \alpha)} \leq \frac{h(u, \alpha)}{h(\alpha, \alpha)}, \quad \text { for } \quad 0<\alpha<u<1,
$$

where, from $(22), h(u, \alpha)=1-u+\phi_{\theta}(u) / \phi_{\theta}^{\prime}(\alpha)$ and $h^{*}(u, \alpha)=1-u+\phi_{\theta^{*}}(u) / \phi_{\theta^{*}}^{\prime}(\alpha)$. Eventually, we have checked that, for all Archimedean family introduced in Table 1, Equation (23) is indeed satisfied when $\theta \leq \theta^{*}$. We then immediately obtain from Proposition 2.12 that each component of $\underline{\mathrm{CTE}}_{\alpha}(\mathbf{X})$ is a decreasing function of $\theta$. Analogously one can prove the result for $\overline{\mathrm{CTE}}_{\alpha}(\mathbf{X})$.

Then, for copulas in Table 1, the multivariate lower-orthant CTE (resp. the upper-orthant $\overline{\mathrm{CTE}}$ ) is non-increasing (non-decreasing) with respect to the dependence parameter $\theta$. In particular, this means that limit behaviors of dependence parameters are associated with bounds for CTE and $\overline{\text { CTE }}$ within the same family of Archimedean copulas.

\footnotetext{
${ }^{6}$ In the book by Nelsen (1999) (Corollary 4.3.5), a geometrical argument is used instead to obtain the distribution function of $(U, C(U, V))$.
} 


\subsection{Behavior of multivariate CTE with respect to risk level}

We now study the behavior of the multivariate lower-orthant and upper-orthant Conditional-Tail-Expectation with respect to risk-level $\alpha$. In particular we obtain the following result.

Corollary 2.3. Consider a d-dimensional random vector $\boldsymbol{X}$ satisfying the regularity conditions.

1. If $\underline{\operatorname{VaR}}_{\alpha}^{i}(\boldsymbol{X})$ is a non-decreasing function of $\alpha$, then $\underline{\mathrm{CTE}}_{\alpha}^{i}(\boldsymbol{X})$ is a non-decreasing function of $\alpha$.

2. If $\overline{\operatorname{VaR}}_{\alpha}^{i}(\boldsymbol{X})$ is a non-decreasing function of $\alpha$, then $\overline{\mathrm{CTE}}_{\alpha}^{i}(\boldsymbol{X})$ is a non-decreasing function of $\alpha$.

Proof: $\quad$ Let us consider the $i$-th coordinate $\underline{\mathrm{CTE}}_{\alpha}^{i}(\mathbf{X})$. From (8) we have

$$
\frac{d}{d \alpha} \underline{\operatorname{CTE}}_{\alpha}^{i}(\mathbf{X})=\frac{K^{\prime}(\alpha)}{1-K(\alpha)}\left[\underline{\operatorname{CTE}}_{\alpha}^{i}(\mathbf{X})-\underline{\operatorname{VaR}}_{\alpha}^{i}(\mathbf{X})\right]
$$

Using Proposition 2.8 the latter expression is non-negative for any level $\alpha \in(0,1)$. The second point of Corollary 2.3 comes down analogously.

The following result proves that assumptions of Corollary 2.3 are satisfied in the large class of $d$ dimensional Archimedean copulas.

Corollary 2.4. Consider a d-dimensional random vector $\boldsymbol{X}$, satisfying the regularity conditions with copula $C$ and survival copula $\bar{C}$.

1. If $C$ is a d-dimensional Archimedean copula, then $\underline{\mathrm{CTE}}_{\alpha}^{i}(\boldsymbol{X})$ is a non-decreasing function of $\alpha$.

2. If $\bar{C}$ is a d-dimensional Archimedean copula, then $\overline{\mathrm{CTE}}_{\alpha}^{i}(\boldsymbol{X})$ is a non-decreasing function of $\alpha$.

Proof: The demonstration of this result comes down from Corollary 2.5 in Cousin and Di Bernardino (2013).

In the univariate setting, the Conditional-Tail-Expectation contains a safety loading i.e., $\mathrm{CTE}_{\alpha}(\mathrm{X}) \geq$ $\mathbb{E}[\mathrm{X}], \forall \alpha \in(0,1)$ (see Section 2.4.3.3 in Denuit et al. (2005)). The safety loading should cover the fluctuations of loss experience. Corollary 2.5 below provides a similar property also for our multivariate lower-orthant ad upper-orthant CTE.

Corollary 2.5. Under assumptions of Corollary 2.3, it holds that

$$
\underline{\mathrm{CTE}}_{\alpha}^{i}(\boldsymbol{X}) \geq \mathbb{E}\left[X_{i}\right], \quad \overline{\mathrm{CTE}}_{\alpha}^{i}(\boldsymbol{X}) \geq \mathbb{E}\left[X_{i}\right]
$$

for all $\alpha \in(0,1)$.

\section{Conclusion and perspectives}

In this paper, we provide two extensions of the Conditional-Tail-Expectation to a multivariate setting. The lower-orthant CTE and the upper-othant CTE can be viewed as natural counterparts of the lowerorthant VaR and upper-orthant VaR introduced in Cousin and Di Bernardino (2013). These measures transform risk vectors into real-valued vectors with the same dimension. The proposed multivariate CTE measures incorporate the entire extreme quadrant part of the underlying distribution function contrary to their multivariate VaR counterparts. They are well-suited for intrinsically multivariate risk problems where, for instance, risks cannot be aggregated together or even compared. We have shown that most properties satisfy by the aforementioned multivariate VaR-s also hold for the studied multivariate CTE-s. In particular, the proposed CTE-s satisfy multivariate version of the positive homogeneity and the translation invariance property, which is not the case for instance for the classical Euler allocation measures recalled in the introduction of this paper. We show that these measures are additive for $\pi$-comonotonic couple of random vectors and we provide an evidence of the sub-additivity property in the independent case. Unsurprisingly, the behavior of the lower-orthant CTE (resp. upper-orthant CTE) with respect to a change in marginal risks or to a change in dependence structure turns to be the same as for the lower-orthant VaR (resp. upper-orthant VaR). 
Extension to discrete distributions can be done by adapting the work by Lee and Prékopa (2012). However this interesting topic goes beyond the scope of the present paper. Another subject of future research should be to compare our multivariate Conditional-Tail-Expectations with existing multivariate generalizations of these measures presented in the introduction, both theoretically and experimentally.

Acknowledgements: This work has been partially supported by the French research national agency (ANR) under the reference ANR-08BLAN-0314-01. Part of this work also benefit from the support of the MIRACCLE-GICC project.

\section{References}

Acharya, V. V., Pedersen, L. H., Philippon, T., and Richardson, M. P. (2010). Measuring systemic risk. FRB of Cleveland Working Paper.

Adrian, T. and Brunnermeier, M. K. (2011). Covar. NBER Workin Paper.

Artzner, P., Delbaen, F., Eber, J. M., and Heath, D. (1999). Coherent measures of risk. Math. Finance, $9(3): 203-228$.

Bargès, M., Cossette, H., and Marceau, É. (2009). TVaR-based capital allocation with copulas. Insurance Math. Econom., 45(3):348-361.

Brownlees, C. T. and Engle, R. F. (2012). Volatility, correlation and tails for systemic risk measurement. working paper.

Burgert, C. and Rüschendorf, L. (2006). Consistent risk measures for portfolio vectors. Insurance: Mathematics Economics, 38(2):289-297.

Cai, J. and Li, H. (2005). Conditional tail expectations for multivariate phase-type distributions. Journal of Applied Probability, 42(3):810-825.

Cai, J.-J., Einmahl, J. H. J., de Haan, L., and Zhou, C. (2013). Estimation of the marginal expected shortfall: the mean when a related variable is extreme. CentER Discussion Paper, 2012-080.

Cascos, I. and Molchanov, I. (2007). Multivariate risks and depth-trimmed regions. Finance and Stochastics, 11(3):373-397.

Chakak, A. and Ezzerg, M. (2000). Bivariate contours of copula. Comm. Statist. Simulation Comput., 29(1):175-185.

Chaouch, M., Gannoun, A., and Saracco, J. (2009). Estimation de quantiles géométriques conditionnels et non conditionnels. J. SFdS, 150(2):1-27.

Chauvigny, M., Devineau, L., Loisel, L., and Maume-Deschamps, V. (2011). Fast remote but not extreme quantiles with multiple factors: applications to solvency ii and enterprise risk management. European Actuarial Journal, 1(1):131-157.

Cousin, A. and Di Bernardino, E. (2013). On multivariate extensions of Value-at-Risk. Journal of Multivariate Analysis, 119(0):32 - 46 .

Denuit, M. and Charpentier, A. (2004). Mathématiques de l'assurance non-vie. Tome 1: principes fondamentaux de théorie du risque. Economica.

Denuit, M., Dhaene, J., Goovaerts, M., and Kaas, R. (2005). Actuarial Theory for Dependent Risks. Wiley.

Di Bernardino, E., Laloë, T., Maume-Deschamps, V., and Prieur, C. (2013). Plug-in estimation of level sets in a non-compact setting with applications in multivariate risk theory. ESAIM: Probability and Statistics, 17:236-256. 
Ekeland, I., Galichon, A., and Henry, M. (2012). Comonotonic measures of multivariate risks. Mathematical Finance, 22(1):109-132.

Embrechts, P. and Puccetti, G. (2006). Bounds for functions of multivariate risks. Journal of Multivariate Analysis, 97(2):526-547.

Hamel, A. H. and Heyde, F. (2010). Duality for set-valued measures of risk. SIAM Journal on Financial Mathematics, 1(1):66-95.

Imlahi, L., Ezzerg, M., and Chakak, A. (1999). Estimación de la curva mediana de una cópula $C\left(x_{1}, \ldots, x_{n}\right)$. Rev. R. Acad. Cien. Exact. Fis. Nat, 93(2):241-250.

Jouini, E., Meddeb, M., and Touzi, N. (2004). Vector-valued coherent risk measures. Finance Stoch., $8(4): 531-552$.

Koltchinskii, V. I. (1997). M-estimation, convexity and quantiles. Ann. Statist., 25(2):435-477.

Landsman, Z. M. and Valdez, E. A. (2003). Tail conditional expectations for elliptical distributions. $N$. Am. Actuar. J., 7(4):55-71.

Lee, J. and Prékopa, A. (2012). Properties and calculation of multivariate risk measures: MVaR and MCVaR. Working paper, Rutgers University.

Mainik, G. and Schaanning, E. (2012). On dependence consistency of covar and some other systemic risk measures. workin paper.

Massé, J.-C. and Theodorescu, R. (1994). Halfplane trimming for bivariate distributions. J. Multivariate Anal., 48(2):188-202.

Nappo, G. and Spizzichino, F. (2009). Kendall distributions and level sets in bivariate exchangeable survival models. Information Sciences, 179:2878-2890.

Nelsen, R. B. (1999). An introduction to copulas, volume 139 of Lecture Notes in Statistics. SpringerVerlag, New York.

Prékopa, A. (2012). Multivariate value at risk and related topics. Annals of Operations Research, 193:4969. $10.1007 / \mathrm{s} 10479-010-0790-2$.

Puccetti, G. and Scarsini, M. (2010). Multivariate momonotonicity. Journal of Multivariate Analysis, 101:291-304.

Rossi, C. (1973). Sulle curve di livello di una superficie di ripartizione in due variabili. Giornale dell'Istituto Italiano degli Attuari, 36:87-108.

Rüschendorf, L. (2006). Law invariant convex risk measures for portfolio vectors. Statistics and Decisions, 24:97-108.

Scaillet, O. (2004). Nonparametric estimation and sensitibity analysis of expected shortfall. Mathematical Finance, 14(1):115-129.

Serfling, R. (2002). Quantile functions for multivariate analysis: approaches and applications. Statist. Neerlandica, 56(2):214-232. Special issue: Frontier research in theoretical statistics, 2000 (Eindhoven).

Tasche, D. (2008). Capital allocation to business units and sub-portfolios: the Euler Principle. Quantitative finance papers, arXiv.org.

Tibiletti, L. (1991). Sulla quasi concavita delle funzioni di ripartizione n-dimensionali - on quasi-concavity of n-dimensional distribution functions. In Atti del XV convegno A.M.A.S.E.S., pages 503-515.

Tibiletti, L. (1995). Quasi-concavity property of multivariate distribution functions. Ratio Mathematica, 9:27-36. 